\title{
Macrophage-derived microRNA-223 Promotes Intestinal Mucosal Barrier Dysfunction via Exosomal Pathways in Inflammatory Bowel Disease
}

\section{Xin CHang}

Naval Medical University https://orcid.org/0000-0002-7623-188X

\section{Yi-hang Song}

Changhai Hospital

\section{Zi-xuan He}

Changhai Hospital

\section{Sheng-bing Zhao}

Changhai Hospital

\section{Jia-yi Wu}

Changhai Hospital

\section{Lun Gu}

Changhai Hospital

\section{Zhao-shen Li}

Changhai Hospital

\section{Can Xu}

Changhai Hospital

\section{Shu-ling Wang}

Changhai Hospital

Yu Bai ( $\sim$ baiyu1998@hotmail.com )

Changhai Hospital

\section{Research}

Keywords: inflammatory bowel disease, macrophage, exosomes, microRNA-223, transmembrane and immunoglobulin domain containing 1

Posted Date: December 15th, 2021

DOI: https://doi.org/10.21203/rs.3.rs-1112924/v1

License: (c) (1) This work is licensed under a Creative Commons Attribution 4.0 International License. Read Full License 


\section{Abstract}

Background \& Aim: Exosomes are effective mediators of cell-to-cell interactions and carry many regulatory molecules, including miRNAs, that can play crucial roles in diverse fundamental biological processes. However, to date, no study has reported macrophage exosomal involvement in the development of inflammatory bowel disease (IBD). This study investigated the specific miRNAs in macrophage-derived exosomes in IBD and the potential molecular mechanism.

Methods: Dextran sulfate sodium (DSS) was used to generate IBD mice. The supernatants of murine bone marrow-derived macrophages (BMDMs) with or without lipopolysaccharide (LPS) were collected for exome isolation and miRNA sequencing. Lentiviruses were used to modify miRNA expression and further investigate the role of macrophage-derived exosomal miRNAs. In vitro, both mouse and human organoids were applied to a Transwell system in co-culture with BMDMs as a cellular IBD-related challenge.

Results: Here, we show that LPS-induced macrophages can release exosomes containing various miRNAs, aggravating IBD. We analyzed miRNA sequencing of macrophage-derived exosomes, and miR223 was selected for further study. In vivo, exosomes with high miR-223 expression contributed to the exacerbation of intestinal barrier dysfunction, which was further verified in both mouse and human colon organoids. Furthermore, time-dependent analysis of the mRNAs of DSS-induced colitis mouse tissue combined with miR-223 target gene prediction was performed to select the candidate gene, and the barrier-related factor TMIGD1 was identified.

Conclusion: Collectively, these data indicated that macrophage-derived exosomal miR-223 played a novel role in intestinal barrier dysfunction by inhibiting TMIGD1 in the progression of DSS-induced colitis.

\section{Introduction}

Inflammatory bowel disease (IBD), a common digestive disease in Western countries, is a group of chronic idiopathic diseases, including ulcerative colitis (UC) and Crohn's disease (CD)[1]. The incidence of IBD in the general population is approximately $0.3 \%$. Due to the increased popularity of the Western lifestyle, the incidence of IBD worldwide is increasing and even catch up to that in developed countries[2]. In addition, in recent years, epidemiological studies have reported that IBD patients are more likely to be young, which could have a significant impact on their quality of life and pose a large medical burden to society $[3,4]$. At present, the pathogenesis of IBD has not been clarified, but several studies have shown that it might be related to defects in the intestinal mucosal barrier and abnormal immune regulation[4]. Due to direct exposure to digestive residues, millions of pathogens and high concentrations of foreign antigens, the intestinal environment is one of the most complex ecosystems in the human body, and an intact intestinal barrier can effectively maintain intestinal homeostasis and prevent luminal substances from entering the body. Several studies have found that the occurrence and development of IBD may be related to the dysfunction of intestinal barriers, thus contributing to the presence of intestinal microorganisms and persistent intestinal inflammation[5, 6]. At the same time, abnormal host defense 
system functions, such as damage to the mucosal barrier and imbalance of the immune system, can also lead to disease occurrence and progression, and intestinal mucosal barrier dysfunction is often accompanied by overexpression of inflammatory factors[7]. Studies have shown that during the pathogenesis of acute colitis induced by dextran sulfate sodium (DSS), the infiltration of a large number of proinflammatory monocytes and other natural immune cells plays an important role. Therefore, research on intestinal mucosal barrier dysfunction caused by abnormal activation of immune cells and the related regulatory mechanism has become a hot topic in the field of IBD.

The innate immune response has been studied extensively in recent years, and macrophages are one of the key players. Polarized macrophages accumulate in the local intestinal segment of IBD lesions and release various proinflammatory or regulatory anti-inflammatory cytokines, which exert biological effects on IBD progression[8]. Newly recruited CD14 and CD11c highly positive monocytes and immature macrophages regulate the differentiation of the immunosuppressive population of resident macrophages $\left(C D 64^{+} H L A-D R^{\text {high }} \mathrm{CD} 14^{\text {low }}\right.$ ) into proinflammatory macrophages, thus promoting the secretion of tumor necrosis factor- $\alpha$ (TNF- $\alpha$ ), interleukin 1- $\beta$ (IL-1 $\beta$ ) and IL-6[9]. In experiments in vitro, epithelial cell apoptosis and epithelial barrier function depend on a well-controlled state of THP-1 cells, and the authors found that the activation of macrophages directly affects IBD development[10]. In addition, the regulation of adaptive immunity by macrophages is mainly reflected in the action on effector $T$ cells and inflammatory cytokines in IBD. Hadis et al. recently showed that intestinal macrophages promote the activation and proliferation of antigen-specific Foxp $3^{+}$regulatory T cells (Tregs) in the intestinal mucosa by secreting IL-10, while knockdown of the TRAF6 gene in CD11 $\mathrm{c}^{+}$monocytes/macrophages resulted in spontaneous Th2 cell-mediated enteritis in mice[11]. The role of macrophages in regulating inflammation-related factors varies: in addition to the proinflammatory effect during activation, macrophages can also secrete protective molecules, such as TGF- $\beta 1$, which binds to Smad7, thus preventing the disruption of intestinal homeostasis[12]. Thus, intestinal macrophages are important players in the inflammatory microenvironment not only for their function in the phagocytosis of pathogens but also for their key role in stimulating the subsequent immune response, secreting a variety of cytokines and acting together with multiple immune cells. Therefore, more efforts should be made to studying the specific role of macrophages in the initiation and development of IBD.

In recent decades, extracellular vesicles (EVs) have been recognized as novel mediators of most cell-tocell interactions[13]. Exosomes (40-150 nm) are produced via the lysosomal/endosomal system, which was discovered by Rose Johnstone in the 1970s[14]. The continuous advances in genome sequencing and proteomic analysis techniques, allowed Valadi et al. to demonstrate in 2007 that exosomes contained bioactive cargo, such as nucleic acids (DNA, mRNA, microRNA (miRNA), and other noncoding RNAs), proteins (receptors, transcription factors, enzymes, and extracellular matrix proteins), and lipids, which can regulate the functions of recipient cells[15]. Moreover, phospholipids, phosphatidylserine, cholesterol, ceramide and other substances contained in the lipid bilayer can maintain the biological activity of the contents by preventing degradation during transport[16]. Therefore, exosomes are considered new carriers that participate in intercellular information exchange. Exosomes from some 
immune cells play an important role in the development of IBD. For example, during neutrophil infiltration, exosomes containing proinflammatory molecules can aggravate double strand breaks in colonic epithelium cells[17]. Dendritic cell-derived exosomes have been shown to be involved in IBD in many studies[18-20]. However, few studies have focused on the role of exosomes derived from macrophages. Yang et al investigated the positive effect of M2 macrophage exosomes on DSS-induced colitis, while the effect of exosomes of M1 macrophages was not determined[21]. Therefore, the function and influence of macrophage-derived exosomes remain to be further investigated.

miRNAs are small noncoding RNAs that can act as regulators of mRNA expression and translational efficiency in most cell types. They can bind to mRNAs and thus lead to mRNA degradation and are the key molecules in exosomes. Cell-derived exosomes contain numerous miRNAs that can be taken up into neighboring or distant cells, thus modulating the function of recipient cells. For example, it was reported that miRNAs encapsulated in exosomes could regulate the signaling process by decreasing the protein expression of targeted mRNAs[22]. Previous studies have shown that there is a change in exosomes when macrophages are activated[23]. Evidence led us to hypothesize that macrophages secrete exosomal miRNAs, which serve as extracellular molecules that can regulate the development of IBD. Here, we report that lipopolysaccharide (LPS)-induced macrophages secrete exosomes containing miR-223 that can be taken up into epithelial cells, both in vitro and in vivo, leading to intestinal barrier dysfunction and promoting the initiation and development of IBD. Furthermore, we explored whether exosomal miR223 affects the development of IBD by repressing its target gene transmembrane and immunoglobulin domain containing 1 (TMIGD1).

\section{Results}

\section{Macrophages were activated during DSS-induced colitis with intestinal mucosal barrier dysfunction.}

The pathogenesis of IBD is not clear, but it may be related to intestinal mucosal barrier defects and immune dysregulation. Weight loss, an increase in DAl and a significant shortening of colon length were observed in the DSS-induced IBD experimental group compared to the control group (Figure 1A-D).

In addition, Alcian blue-periodic acid-Schiff (AB-PAS) staining was used to detect mucous secretion in the intestines of mice to indirectly evaluate intestinal mucosal barrier integrity, and the mice in the DSS group showed a significant decline in intestinal mucosal barrier integrity (Figure 2A). Correspondingly, in the Fluorescein Isothiocyanate-Dextran (FITC-D) and flora translocation experiments, the mice in the DSS group showed a statistically significant increase (Figure 2B). Moreover, the DSS group showed a significant decrease in the levels of detected intestinal mucosal barrier-related molecules, such as intestinal ZO-1 and Occludin (Figure 2C-D). The increased rate of bacterial translocation in the DSS groups indicated indirect damage to the intestinal barrier (Figure 2E). These experimental results indicated that there were intestinal mucosal barrier defects in the IBD mouse model.

The expression levels of IL-1 $\beta$, TNF- $a$, IL- 6 and IL-18 in the intestinal tissue of the DSS group mice were significantly higher than those in the control group mice (Figure $3 A$ ). Using H\&E staining, we further 
evaluated the destruction of the pathological structure of the colon of IBD mice (Figure 3B). By immunofluorescence, we examined the expression of intestinal macrophages and found that the number of macrophages in the DSS group was significantly increased compared to that in the control group (Figure 3C).

\section{Activated macrophage-derived exosomes aggravated colon organoid apoptosis.}

In recent years, exosomes have become a research hotspot as vehicles for intercellular communication. Previous studies have shown a significant increase in the number of exosomes secreted by macrophages after activation. However, the roles of these exosomes remain unclear. Here, we used colon organoids to model organ development and disease with more sample accessibility and fewer ethical concerns. Both mouse and human colon organoids were cultured to further explore the role of exosomes derived from activated macrophages, especially in the intestinal mucosal barrier.

Mouse-derived organoids were passaged three times to maintain their stability and then cocultured with macrophages. When cocultured with LPS-activated macrophages, the growth rate of mouse colonic organoids was observed to be significantly decreased compared to coculture with resting macrophages (Figure 4A). Furthermore, the expression of the barrier marker E-cadherin was detected by immunofluorescence (Figure 4B). Human-derived colonic organoids were cultured by collecting tissues from four normal human colons, and the same result was observed in human-derived colonic organoids. To assess whether this effect is mediated by macrophage-derived exosomes, we used GW4869, an inhibitor of exosome production. After treatment with GW4869, the disruption of the intestinal mucosal barrier and inhibition of organoid growth were relieved (Figure 4C-D).

\section{Macrophage-derived exosomes were isolated and identified.}

For the accuracy of the miRNA sequencing results, exosomes were all extracted from primary macrophage cells. In the experimental group, macrophages were activated by LPS stimulation. To identify the macrophage subsets, cells were isolated from mouse bone marrow tissue and later induced by M-CSF colony-stimulating factor, washed in PBS, and stained with fluorochrome-conjugated antibodies against the cell-surface antigens F4/80, CD11b, and CD68. Primary macrophages were verified by flow cytometry analysis of the cytokines F4/80 and CD11b. The identification of double stained cells demonstrated that the primary macrophages were successfully induced. In the LPS group, CD68 expression in macrophages was significantly increased according to flow cytometry (Supplementary Figure 1A). Furthermore, inflammatory factors such as TNF-a, IL-6, and IL-1 $\beta$ were further detected in isolated macrophages, and the expression of these factors in the experimental group was significantly higher than that in the control group (Supplementary Figure 1B).

Exosome isolation was performed by trained staff, and exosomes were successfully extracted from cell culture supernatants with QIAGEN kits and analyzed by the NanoSight and Q-NANO microfluidic systems

to determine the size and concentration of exosomes (Figure 5A-B). The purity, quality, and morphology of the exosomes were analyzed using negative-staining TEM (Figure 5C). As expected, ex vivo 
fluorescence imaging showed that macrophage-derived exosomes could enter enterocytes (Figure 5D). In addition, we used CD9 exosomal markers for protein level verification. (Figure 5E). The verified exosomes were sequenced for miRNA analysis, and differentially expressed genes were identified. The results indicated that miR-223 had a significantly higher expression in the macrophage activation group, and the expression of miR-223 was verified in vitro. The expression of miR-223 in the experimental group showed a statistically significant increase in both macrophages and exosomes compared with that in the control group (Figure 5F). We identified 325 differentially expressed miRNAs, 127 of which were upregulated. We selected the 15 most significantly changed miRNAs as candidates. MiR-223 is a myeloid cell-specific miRNA, and its abundance and function in inflammatory diseases are evident (Figure 5G).

\section{Activated macrophagederived exosomes carrying miR-223 promoted the progression of colitis in mice treated with DSS.}

To further investigate the effect of exosomal miR-223 on disease development during IBD, exosomes were extracted from different processed RAW264.7 cells and injected into mice receiving 3\% DSS for 7 days. Disease severity was evaluated by the presence of sustained weight loss (Figure 6A), bloody diarrhea (Figure 6B) and shortened colon length (Figure 6C). Compared with mice in the DSS+exosomes ${ }^{\text {miR-223 agomir NC }}$ group, mice in the DSS+exosomes ${ }^{\text {miR-223 agomir }}$ group exhibited aggravated weight loss, an increase in disease activity score, and a significant shortening of colon length, and the differences between the two groups were statistically significant. There was no significant difference between the DSS+exosomes ${ }^{\mathrm{miR}-223 \text { antagomir }}$ group and its negative control group. In terms of DSS, the expression levels of TNF- $a, \mathrm{IL}-18$, and IL-10 in the intestinal tissues of mice in the DSS+exosomes $^{\text {miR-223 agomir }}$ group were significantly higher than those in the DSS+exosomes $^{\text {miR-223 }}$ agomir NC group (Figure 6D). Then, we further evaluated the impact of miR-223 on the colonic pathological structure of the DSS+exosomes ${ }^{\mathrm{miR}-223}$ agomir group by H\&E staining, and we found that high expression of miR-223 in exosomes could promote the destruction of colonic tissue and inflammatory infiltration (Figure 6E).

\section{Exosomes with high expression of miR-223 promoted the destruction of the intestinal mucosal barrier in an IBD mouse model and organoids.}

The expression of ZO-1 and Occludin-1 at both the RNA and protein levels in the colonic tissue of mice in the DSS+exosomes ${ }^{\text {miR-223 agomir }}$ group was significantly lower than that in the DSS+exosomes ${ }^{\text {miR-223 }}$ agomir NC group (Figure 7A-B); we further detected the intestinal mucus secretion of the mice in the 6 different groups by AB-PAS staining and the expression of E-Cadherin in the intestinal tract by immunofluorescence (Figure 7C-D). The number of goblet cells reflects the state of the intestinal mucus barrier. Figure 7D shows that the number of goblet cells significantly decreased in the DSS group compared to the control group. However, the further deterioration in the DSS+exosomes ${ }^{\text {miR-223 agomir }}$ group indicated that exosomes with high miR-223 expression can aggravate barrier dysfunction (Figure 7E). Immunofluorescence showed that the expression of the intestinal mucosa-related molecule E- 
cadherin significantly decreased in the mice in the DSS+exosomes ${ }^{\text {miR-223 agomir }}$ groups compared to the corresponding NC group (Figure 7F). Furthermore, TEM was used to observe desmosomes, epithelial microvilli, and tight junctions in the mouse colon and to analyze the integrity of the colon tissue. Compared with that in the control group, the colonic tissue of mice in the DSS group showed a decrease in the number of desmosomes, distribution of intestinal villi and tight connection twists, which were also

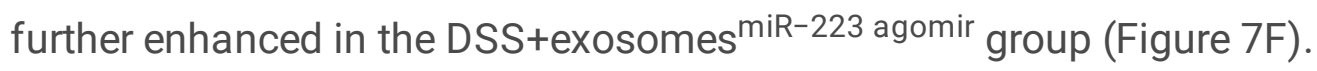

Next, stably transfected cell lines were activated with LPS medium and cocultured with cells and colonoids (Supplementary Figure 2). We found that human organoid development was negatively related to the expression of miR-223 (Figure 8A). Compared to that in the control group, the number of PCNApositive fluorescence points in the DSS group was significantly reduced, suggesting a significant decrease in growth; at the same time, the number of E-cadherin-positive fluorescence points was significantly decreased, which demonstrated that the intestinal mucosal barrier was also significantly decreased, and the above changes in the LPS group were further aggravated in the LPS+miR-223 agomir group (Figure 8B). Moreover, we investigated the effect of macrophage-derived exosomes containing miR223 on the mouse colonic organoids, and we found that miR-223-containing macrophage-derived exosomes also aggravated the destruction of the intestinal mucosal barrier, affecting their growth during this period (Figure 8C). Figure 8D shows that the number of macrophages significantly increased in the DSS group compared to the control group, similar to the 2 groups analyzed previously. However, the number of macrophages was further increased in the DSS+exosomes ${ }^{\text {miR-223 agomir }}$ group, which suggests a positive loop between miR-223 and macrophages.

\section{The target gene of miR-223 in intestinal tissue was investigated.}

The relevant mRNA sequencing data of IBD mice were obtained from NCBI, and the mRNA expression data set was screened and analyzed. The time-dependent expression of the mRNA transcriptome of the IBD mouse model was analyzed in a time series study. A total of 218 genes were identified when looking for gene transcripts that had the opposite expression trend to miR-223 expression, with their expression continuously decreasing during the modeling process (the first 7 days) and continuously increasing after the removal of the modeling conditions (the following 7 days). A total of 437 downstream target genes of miR-223 were identified in the miRBase database, and a total of 2 genes were obtained after intersecting these genes with the screening results of the previous biomarker analysis (Figure 9A-B). Among them, TMIGD1 is specifically expressed in the intestine, and previous studies have shown that TMIGD1 can be significantly expressed at lower levels in human intestinal cancer tissues. We analyzed the binding rate, and the absolute values of minimum free energy were more than 20 (Figure 9C). A TMIGD1 wild-type plasmid and a 3'UTR mutant plasmid were constructed. By detecting the relative luciferase activity after cotransfection of miR-223 analog (or NC) and wild-type (or mutant) vector plasmid in HEK293 cells, the regulatory effect of miR-223 on TMIGD1 was confirmed (Figure 9D). To further investigate the relationship between miR-223 and TMIGD1, we detected the expression of the target gene TMIGD1 in the tissue of mice from the 6 groups mentioned above and obtained the same result (Figure 9E-F). 


\section{The expression of TMIGD1 in the intestinal tissues of patients with IBD was evaluated.}

Patients with IBD were recruited from the endoscopy center of Changhai Hospital from September 2019 to December 2019, clinical data were collected, and colon tissue samples were collected during endoscopy. All of work described has been carried out in accordance with The Code of Ethics of the World Medical Association (Declaration of Helsinki) for experiments involving humans. informed consent was obtained for experimentation with human subjects. Patients were evaluated by H\&E staining and the Mayo score. Furthermore, the expression of TMIGD1 was detected by histochemical immunostaining and further analyzed according to the patients' disease condition. As shown in Figure 10A, the expression of TMIGD1 was negatively correlated with disease severity, which was evaluated according to the Mayo score. Then, to further explore the function of TMIGD1, cells were transduced with TMIGD1overexpressing lentivirus and siRNA-mediated TMIGD1-knockdown lentivirus to obtain TMIGD1-Up and TMIGD1-Down intestinal epithelial NCM460 cells, respectively, along with negative control cells (Figure 10B). The TEER measurement module experiment showed that the permeability of the NCM460 intestinal epithelial cell line was significantly increased in the TMIGD1 inhibitory group (Figure 10C). Subsequently, by immunofluorescence, we found that the expression of ZO-1 was decreased in TMIGD1-knockdown NCM460 intestinal epithelial cells (Figure 10D).

\section{Discussion}

Various studies have illustrated that macrophages play an important role in the pathogenesis of IBD. There are several major findings in our research. First, we revealed that exosomes derived from activated macrophages modulate the intestinal mucosal barrier and contribute to the initiation and development of IBD. Next, we isolated exosomes from activated BMDMs and performed miRNA sequencing analysis. We found 325 differentially expressed miRNAs, 127 of which were upregulated, and miR-223 was one of the most important factors. In vivo studies showed that activated macrophage-derived exosomes carrying miR-223 promote the progression of colitis in mice treated with DSS. Exosomes with high expression of miR-223 were further found to contribute to dysfunction of the intestinal mucosal barrier in both mouse and human colonic organoids. Relevant mRNA sequencing data of IBD mice were obtained from NCBI, and 2 genes (TMIGD1 and HLF1) were obtained after intersecting with the screening results of the previous biomarker analysis. Then, we proved that miR-223 contained in macrophage-derived exosomes can target the barrier-related molecule TMIGD1 in intestinal epithelial cells, thus promoting the development of IBD. All of these data indicated that macrophage-derived exosomes containing miR-223 play a novel role in intestinal barrier dysfunction in the progression of DSS-induced colitis by inhibiting TMIGD1(Supplementary Figure 3).

Patients with IBD have increased intestinal mucosal permeability due to intestinal mucosal barrier dysfunction, which in turn causes flora displacement and intestinal microecological dysbiosis. Both bacterial components and necrotic apoptotic intestinal epithelial cells can produce large amounts of LPS, which recruits and activates natural immune cells, especially Toll-like receptor macrophages, and is involved in the inflammation and development of IBD[24]. Previous studies have shown that IL-10 inhibits 
macrophage activity, glucose uptake and glycolysis after stimulation of macrophages by LPS and promotes oxidative phosphorylation by suppressing the mTOR signaling pathway, thereby maintaining intestinal homeostasis[25]. Compared with that of normal mucosal lamina propria macrophages, the number of intestinal mucosal macrophages in the colonic tissues of patients with active IBD was significantly increased, and these macrophages tended to be more activated, suggesting that intestinal macrophages played an important role in the development of IBD[26]. Consistent with these previous studies, our findings also confirmed the significant role of macrophages in IBD.

Exosomes are considered new mediators of intercellular genetic transfer and intercellular information carriers. Exosomes play an important role in the development and regression of intestinal diseases, especially IBD. Macrophages can also secrete exosomes after stimulation, such as bacterial infection, innate immune system activation, induction of a proinflammatory response, and thus participate in the development of IBD[27]. Others have demonstrated that macrophage-derived EVs can rescue intestinal stem cells and enhance the survival rate of enterocytes after radiation injury through the regulation of WNT function[28]. Our study also validated the key role of macrophage-derived exosomes in IBD.

The concept that exosomal miRNAs can be transferred to other cell types is supported by many systems in vitro and in vivo. In 2021, in vitro data demonstrated that endothelial cell-derived EVs can target vascular cells[29]. Then, Ying et al. showed that adipose tissue macrophage-derived exosomal miRNAs can modulate insulin sensitivity by transferring miRNAs to insulin target cells[30]. With respect to the intestinal barrier, many studies have shown that functional miRNAs have a critical role in the intestinal barrier. Liu et al. reported that miR-381-3p inhibition enhanced intestinal epithelial proliferation and restored intestinal barrier function in intestinal $\mathrm{I} / \mathrm{R}$ injury by suppression of its target gene nuclear receptor-related protein 1 (nurr1)-mediated proliferative pathway[31]. UC patients have higher expression of miR-24 in colonic biopsies and blood samples, which leads to a decrease in barrier integrity[32]

MiRNAs encapsulated in exosomes are selectively and actively loaded, which is a key crosstalk mechanism in IBD regulation[33-35]. In many studies, receptor-binding proteins, known as membranous proteins, facilitate the selective packaging of miRNAs in many diseases[36, 37]. In addition, because exosomal cargo is protected against degeneration, miRNAs in exosomes can exert more influence than single miRNAs[38, 39]. Furthermore, exosomal membranes enable loaded miRNAs to cross many barriers, such as the blood-brain barrier, opening the possibility that miRNAs loaded in exosomes may be an effective drug delivery system[40]. Most of the current studies on exosomal miRNAs in IBD are biomarker screening research $[41,42]$. Few of them are targeting microbiota. Therefore, in-depth exploration of the specific exosomal miRNAs derived from macrophages responsible for intestinal dysbiosis phenotypes is rare.

Secreted EVs were harvested from macrophages under different conditions by several rounds of centrifugation. We identified these EVs as exomes mainly with a diameter between 20 and 150 nanometers by electron microscopy, NanoSight analysis, and measurement of the exosomal membrane marker CD9. Then, we observed strong differential expression of miR-223 when macrophages were 
treated with LPS. Taken together, these experiments show that macrophages produce miRNA-containing exosomes and that the miRNA cargo can be directly transferred into enterocytes to affect the intestinal barrier in vitro. Our in vivo studies are consistent with this concept and show that treatment of colitis mice

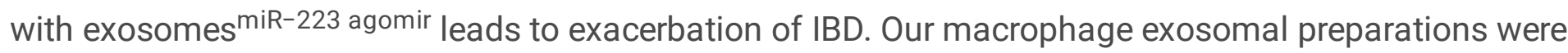
obtained using published techniques and reliable commercial kits, and vesicle preparations were characterized in detail with respect to the expression of reported exosomal protein markers. In particular, we analyzed the remaining medium after exosomes had been isolated and did not detect exosomal membrane markers. It is clear that our extracted exosomes are miRNA-containing macrophage-derived $\mathrm{EVs}$, and based on the consistent results of the characterization of these vesicles with the published literature, we think that it is reasonable to refer to them as macrophage-derived exosomes.

We performed miRNA sequencing of the control and LPS-induced exosomes and identified miR-223 as a highly expressed candidate of interest. It has been reported that miR-223 is a hematopoietic cell-specific miRNA that is preferentially expressed in myeloid cells[43,44]. Various studies have demonstrated its potential as a cargo of immune cell-derived exosomes in intestinal inflammation and barrier dysfunction. $\mathrm{Li}$ et al. demonstrated that mast cell-derived miR-223 had a significant inhibitory effect on intestinal epithelial permeability[45]. MiR-223 has also been characterized as a proinflammatory mediator between the IL-23 pathway and the intestinal barrier in trinitrobenzene sulfonic acid-induced colitis mice[9]. However, Neudecker et al. showed that knocking out miR-223 exacerbated myeloid-driven experimental colitis, which was reversed by nanoparticle-mediated overexpression of miR-223[46]. In our studies, oneweek treatments of colitis mice with different exosomes has variable effects on disease development. Aggravated disease was observed not only in vivo but also in human and mouse organoids cocultured with miR-223 agomir RAW264.7 induced by LPS.

MiR-223 is a popular miRNA not only in IBD but also in other inflammatory diseases. Previous studies have shown that miR-223 plays a positive role in immunosuppression regulation and myeloid differentiation[23]. For example, during lung inflammation, miR-223 suppresses macrophage activation and decreases inflammation through inhibition of NLRP3 inflammasome activation[47]. MiR-223-NLRP3 interactions have an essential effect on other inflammatory diseases or different states of infections. In granuloma formation, miR-223 decreased inflammasome and IL-1 $\beta$ pathway activation[48]. In another study, liver inflammation and fibrosis spontaneously resolved by inducing proinflammatory macrophages into a restorative phenotype via the NLRP3/miR-223 axis[49]. Enhanced delivery of miR-223 can dampen acute lung injury through repression of PARP-1 [50]. During intestinal inflammation, miR-223 can alleviate inflammation by inhibiting the NLRP3 inflammasome[9].

However, different conclusions have been reached in terns if the role of miR-223 in intestinal barrier function. Li et al. demonstrated that mast cell-derived exosomes damaged the intestinal barrier, which was attributed to functional miRNAs being transferred from HMC-1 cells into intestinal epithelial cells, leading to the inhibition of tight junction-related protein expression[51]. In the trauma healing process, miR-223 contained in plasma exosomes was confirmed to act as a protective immune-barrier, and its levels were increased in healing fractures[52]. Therefore, as a neutrophil-related or myeloid-regulated 
gene, miR-223 is associated with restored intestinal dysbiosis. However, in terms of its role in the intestinal mucosal barrier, notwithstanding its negative effect, the precise mechanism is still unclear. For greater certainty of the downstream target genes, we selected downstream target genes of miR-223 in a miRNA target prediction database. In subsequent research, we downloaded the intestinal tissue mRNA expression data of mice that were subjected to DSS for 7 days and restored to normal water for 7 days. The expression of miR-223 was positively associated with the severity of IBD and negatively correlated with target mRNA expression. Therefore, the expression of target mRNA should decrease during the first seven days of disease modeling and should increase during the last seven days of recovery. The mRNA expression data were sorted via time-dependent analysis. Additionally, selected mRNAs that were consistent with the expected expression trend and candidate target mRNAs from the database were intersected to obtain the two final target mRNAs. Finally, according to the predicted interaction score and the negative effect of miR-223 on intestinal barrier function, we chose, and further verified, TMIGD1 as a target gene of miR-223 for further study.

TMIGD1 is not a very well-studied gene; however, it is known to be specifically expressed in intestinal tissue and epithelial cells[53]. TMIGD1 is a glycoprotein located on the cell surface and consists of one or several immunoglobulins, a single transmembrane domain and an intracellular C-terminus. Previous studies have shown that TMIGD1, as an Ig-CAM, is able to bind to different ligands through a foreign fragment to form a linking structure to stabilize the cell membrane structure[53, 54]. In addition, it can strengthen the structural barrier formed by the cell membrane and epithelial cells and improve the resistance of epithelial cells to extracellular adverse factors and the maintenance of homeostasis[55]. Previous research has found that TMIGD1 expression is extremely downregulated in the tissue of experimental colon cancer mice [56]. In a recent study, genome-wide expression data analysis showed that there is a critical downregulation of TMIGD1 expression in the tissue of CD patients[57], which indirectly validates our time-dependent analysis. We observed intestinal barrier function under differential expression of TMIGD1 to further investigate its function.

In summary, we found that LPS-activated macrophages secreted miR-223-containing exosomes. While treatment with miR-223 agomir exosomes led to in vivo and in vitro intestinal barrier dysfunction, treatment with miR-223 antagomir exosomes did not show remission. However, it is unlikely that miR-223 is the sole explanation for the differences in disease development in exosome-treated mice, as there are most likely other miRNAs within these exosomes that act synergistically. In addition, it is possible that multiple miRNAs within the macrophage-derived exosomes could work in a coordinated way to induce the intestinal dysfunction that we observed. Future studies are necessary to identify the full set of LPSinduced exosomal miRNAs causing negative effects on the intestinal barrier. Furthermore, the differences in miR-223 function reported in the literature are a good reminder that the same gene in different cells involved in different processes might vary widely even in the same disease. This is the first time that TMIGD1 has been discussed as a protective gene in IBD. Further deletion of miR-223 in macrophages or complementing TMIGD1 in the epithelium could be a promising cure for IBD. 


\section{Conclusions}

In conclusion, the data in this paper provide a strong molecular link between miR-223 and IBD by exploring the role and mechanism of intestinal macrophages in IBD. Our results further indicated that macrophage-derived exosomes containing miR-223 play a novel role in intestinal barrier dysfunction by inhibiting TMIGD1 in the progression of DSS-induced colitis. These factors may provide a regulatory strategy for IBD cure in the future.

\section{Materials And Methods}

\section{Animals and ethics}

Male C57BCLA mice aged 7 weeks (weighing $20 \mathrm{~g}$ ) were purchased from the Experimental Animal Center of Changhai Hospital Animal Experimental Center. All animal experimental procedures were approved by the Animal Research Ethics Committee of Changhai Hospital and conformed to the Chinese National Institute of Health Guide for the Care and Use of Laboratory Animals.

\section{Induction of colitis and treatment}

Acute colitis was induced by administering water supplemented with $3 \%(\mathrm{wt} / \mathrm{vol})$ DSS to male C57BCLA mice aged 7 weeks. Mice were weighed daily and observed, and the disease activity index (DAI) was evaluated based on weight loss, diarrhea, and bleeding. Mice were sacrificed on day 7 , and the colon length was measured. Part of the colon tissue was collected and stored at $-80^{\circ} \mathrm{C}$ for protein and RNA extraction, and another part of the colon tissue was fixed in $4 \%$ paraformaldehyde acid and embedded in paraffin for pathological sectioning, hematoxylin and eosin (H\&E) staining and immunohistochemistry experiments.

\section{Macrophage generation}

The femur was obtained from sacrificed C57BCLA mice, washed with 10\% penicillin-streptomycin wash buffer twice, incubated in 75\% ethanol for 10 min, and then placed in Dulbecco's modified Eagle's medium (DMEM). Next, the bones were cut at each end, the marrow cavity was exposed, and the bone was washed with macrophage cell culture medium (DMEM supplemented with $10 \%$ fetal bovine serum (FBS), $30 \mathrm{ng} / \mathrm{mL}$ murine macrophage colony-stimulating factor (M-CSF) and 1\% penicillin-streptomycin). Then, the bone marrow cells were resuspended and cultured in a $37^{\circ} \mathrm{C}$ incubator with $5 \% \mathrm{CO}_{2}$. Refresh the culture medium every 3 days. Usually, marrow-derived macrophages (BMDMs) mature until day 7.

\section{Cell treatment and Exosome isolation}

Usually, marrow-derived macrophages (BMDMs) mature until day 7. The cells were either stimulated with LPS (100 ng/mL, Sigma-Aldrich, St. Louis, MO, USA) or left untreated. The culture medium was changed to exosome-free culture medium, and the cells were harvested after stimulation for $24 \mathrm{~h}$. Exosome 
isolation was performed with an exosome isolation kit (Invitrogen, USA) following the manufacturer's instructions.

Exosomes derived from BMDMs were isolated with exosome isolation kits (QIAGEN, USA) according to the manufacturer's instructions.

Then, total RNA was extracted from BMDM-derived exosomes for small RNA sequencing. Exosomes for in vitro experiments were harvested by differential centrifugation. The detailed steps are listed as follows. FBS free of exosomes was purchased from SBI. BMDMs were cultured in medium with $10 \%$ exosome-free FBS. After $24 \mathrm{~h}$, the cell culture medium was collected, and exosomes were isolated from the supernatant by differential centrifugation at $300 \times \mathrm{g}$ for $10 \mathrm{~min}, 2000 \times \mathrm{g}$ for $15 \mathrm{~min}, 12,000 \times \mathrm{g}$ for $30 \mathrm{~min}$, and $100,000 \times \mathrm{g}$ at $4^{\circ} \mathrm{C}$ for $8 \mathrm{~h}$ to remove floating cells and cellular debris and isolate the exosomes.

\section{Exosomal miRNA sequencing}

miRNA components in either untreated control macrophage exosomes $(n=3)$ or LPS-induced macrophage exosomes were profiled by small RNA sequencing analysis (Illumina). miRNA-seq reads were qualityassessed with FastQC and MultiQC, trimmed with Trimmomatic 0.30 and subsequently aligned with NovoAlign to all mouse miRNA hairpin sequences in release 21 of miRBase. The resulting alignments were tabulated with a Python script to generate per-miRNA counts.

\section{Establishment of RAW 264.7 macrophage with stably expression relative miRNA}

RAW 264.7 macrophages cell line was obtained from the National collection of Authenticated Cell Cultures (Shanghai, China) and cultured in DMEM medium containing $10 \%$ FBS, penicillin $(100 \mathrm{U} / \mathrm{mL})$ and streptomycin $(100 \mathrm{mg} / \mathrm{mL})$, and maintained at $37^{\circ} \mathrm{C}$ in a humidified atmosphere of $5 \% \mathrm{CO}_{2}$ humidified atmosphere chamber.

Lentivirus purchased from Sunbio (Shanghai, Chian) was used to establish stable miR-223-knockout, overexpression and corresponding negative control (NC) macrophage strains. Cells were divided into 4 groups: 2 group was added with $10 \mathrm{~mL}$ cell medium containing knockout and overexpression virus and the other group was added with $10 \mathrm{~mL}$ empty vector virus solution. The multiplicity of infection was 15 . After $8 \mathrm{~h}$ of cell culture, the culture medium was refresh with complete cell medium. After $48 \mathrm{~h}$ of infection and cell growth, positive clones were screened by using a complete culture medium containing 4.0 $\mathrm{mg} / \mathrm{mL}$ puromycin for 3 generations. Then the cell with good condition can be consider as stable strain. The supernatants of stable strain expressing miR-223 agomir NC, miR-223 agomir, miR-223 antagomir $\mathrm{NC}$, and miR-223 antagomir were collected to extract exosomes, and the extracted exosomes were injected intraperitoneally. Stable transfection cell lines also seeded in the transwell system, co- culturing with organoids, as described below.

\section{Exosome isolation from macrophage strains and their treatment on mice}


The following 6 groups were established to observe the effect of exosomal miR-223 on the progression of IBD: control; DSS, in which mice had access to $3 \%$ (wt/vol) DSS; DSS+exosomes ${ }^{\text {miR-223 agomir-NC, in which }}$ mice had access to DSS and were injected with miR-223 agomir NC exosomes; DSS+exosomes ${ }^{\text {miR-223 }}$ agomir, in which mice had access to DSS and were injected with miR-223 agomir exosomes;

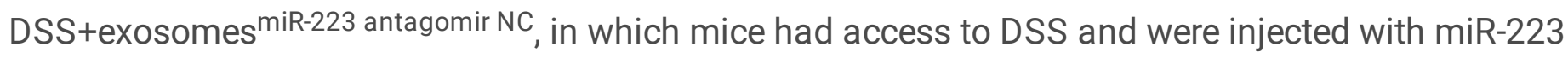

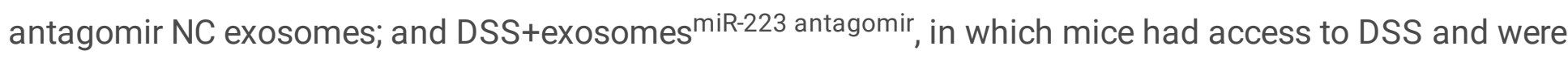
injected with miR-223 antagomir exosomes. Exosomes from different stable macrophage strains were peritoneal injected to the model mice receiving DSS from day 2 to day $7,10^{8}$ every day.

\section{Colon organoid culture and treatment}

Intestinal tissues from mice and humans were freshly obtained. The tissue was digested with $2 \mathrm{mmol} / \mathrm{L}$ EDTA (Gibco, USA) for $30 \mathrm{~min}$ and $5 \mathrm{mmol} / \mathrm{L}$ EDTA (Gibco, USA) for $30 \mathrm{~min}$ twice at $4^{\circ} \mathrm{C}$ on a roller. The tissue was filtered and $10 \mathrm{~mL}$ of $0.1 \%$ BSA (Solarbio, China) was added to neutralize the EDTA and obtain the crypts. After isolation, crypt units were plated to a density of 200 crypts per well in a $40-\mu \mathrm{L}$ droplet of Matrigel mixed with IntestiCult media. Crypt-containing Matrigel droplets were overlaid with IntestiCult complete organoid media (Stemcell Technologies, Vancouver, BC) supplemented with penicillin/streptomycin.

The 4 stable macrophage strains (miR-223 agomir NC, miR-223 agomir, miR-223 antagomir NC, and miR223 antagomir) mentioned above were plated on up-side of transwell insert membrane for a minimum incubation of $12 \mathrm{~h}$ with LPS $(100 \mathrm{ng} / \mathrm{m})$ stimulation. After cell attachment rate is about $60 \%$, cocultured with mouse and human organoids in the presence of LPS $(100 \mathrm{ng} / \mathrm{m}$, refresh every $24 \mathrm{~h})$ to verify the effect of exosomes on the progression of IBD in vitro.

\section{Transcriptome sequencing data and identification of target genes}

GSE1543 and GSE16523 (mRNA profiling data sets based on Affymetrix Human Genome U133 Plus 2.0 Array)-consisting of gene expression data of mouse colon tissues from molding until day 7 to remission until day 14-were obtained from the Gene Expression Omnibus (GEO) database. This study extracted the data on days $0,4,7,10$ and 14 . The raw data of each mRNA were normalized and analyzed by the $R$ package limma and the RobustRankAggreg package. To identify the genes or gene sets coregulated in a time-dependent manner (temporal expression profiles), the software program "Short Time-series Expression Miner" (STEM, version 1.3.11, http:// www.cs.cmu.edu/\$jernst/stem/) was used. The main advantage of STEM is that it implements a novel method for clustering short time series expression data, which can distinguish between real and random patterns. To reduce the redundancy, the group was defined as temporal expression profile $n(n=1,2,3 \ldots n)$ according to the corresponding $P$ values. Genes belonging to profile $n$ were fellow named temporal expression profile genes $n$ (TEPGs).

\section{Collection and scoring of human samples}


Clinical tissue biopsies were collected from the Digestive Endoscopy Center of Changhai Hospital, an open-access academic tertiary endoscopy center. The protocol of this study was carried out according to the principles of the Declaration of Helsinki and approved by the Medical Ethics Committee in Shanghai Changhai Hospital, Shanghai, China. Written informed consent was obtained from all of the participants before enrollment. The severity of disease ranging from a mild/moderate to severe state was evaluated according to the improved Mayo scoring system. The tissue was fixed in $4 \%(\mathrm{w} / \mathrm{v})$ paraformaldehyde overnight or stored at $-80^{\circ} \mathrm{C}$ for further experiments.

\section{RNA extraction and real-time polymerase chain reaction (RT-PCR)}

Total RNA was isolated using TRIzol reagent (Invitrogen, USA) and quantified by measuring the ratio of the absorbance at 260 and $280 \mathrm{~nm}$ using a NanoDrop 2000 spectrophotometer (Thermo Scientific, USA). Then, RNA was converted to complementary DNA (cDNA) using a Thermo Scientific Revert Aid First Strand cDNA Synthesis Kit (Thermo Scientific, USA) according to the manufacturer's instructions. Furthermore, reverse transcription PCR was performed using SYBR Green QPCR Master Mix (TaKaRa, Japan). The reaction conditions comprised an initial denaturation at $95^{\circ} \mathrm{C}$ for $30 \mathrm{~s}$, followed by amplification for 35 cycles at $95^{\circ} \mathrm{C}$ for $5 \mathrm{~s}$ and $60^{\circ} \mathrm{C}$ for $20 \mathrm{~s}$. The primers were as follows: Claudin I, sense 5'- CCAGGTACGAATTTGGTCAG G -3' and antisense 5'- TGGTGTTGGGTAAGAGGTTGT -3'; Occludin, sense 5'- CCAGGTACGAATTTGGTCAGG -3' and antisense 5'- TGGTGTTGGGTAAGAGGTTGT -3'; ZO-1, sense 5'- TCATCCCAAATAAGAACAGAGC -3' and antisense 5'- GAAGAACAACCCTTTCATAAGC -3'; TNF-a, sense 5'- CAGGCGGTGCCTATGTCTC -3' and antisense 5'- CGATCACCCCGAAGTTCAGTAG -3'; IL-1 $\beta$, sense 5'- CGAATGCCACCTTTTTGACAGTG -3' and antisense 5'- TAGATGCTCTCATCAGGATAG -3'; IL-18, sense 5'GAAATGCCACCTTTTTGACAGTG -3' and antisense 5'- CTCCAGCATCAGGACAAAGAAAGCCG -3'; IL-10, sense 5'- GGTTGTCGTCTCATTCTGAAAGA -3' and antisense 5'- GGTAGAGGACCCAAGTTCGTTAAGA -3'; IL-6, sense 5'- ATGAACTCCTTCTCCACAAGC $-3^{\prime}$ and antisense 5'CTACATTTGCCGAAGAGCCCTCAGGCTGGACTG -3'; and GAPDH, sense 5'CCATTTGATGTTAGCGGGATCTC -3' and antisense 5'- TGGTCTACATGTTCCAGTATGACT - 3 '.

\section{Protein extraction and western blot analysis}

Intestinal frozen tissues were thawed at $4^{\circ} \mathrm{C}$, and protein was extracted with RIPA buffer (Biyuntian, China) according to the manufacturer's instructions. Nuclear protein from NCM460 cells was obtained by sonication of nuclear pellets followed by centrifugation. Equal amounts of protein were loaded onto sodium dodecyl sulfate-polyacrylamide gel electrophoresis (SDS-PAGE) gels. After electrophoresis, polyvinylidene fluoride (PVDF) membranes were blocked with $5 \%$ nonfat dry milk in TBS-T (20 mM Tris/ $\mathrm{HCl} \mathrm{pH} \mathrm{7.2,} 150 \mathrm{mM} \mathrm{NaCl}$, and $0.1 \%$ Tween-20) and incubated overnight with different primary antibodies. The dilution concentration was as follows: anti-CD9 (1:500, Abcam, USA), anti-Occludin (1:500, Thermo Scientific, USA), anti-ZO-1 (1:125, Thermo Scientific, USA), anti-E-cadherin (1:1000, Cell Signaling Technology, USA), and anti-TMIGD1 (1:200, Proteintech, China). Next, the membranes were incubated with peroxidase-conjugated anti-mouse IgG $(1: 2,500$; Thermo Scientific, USA) or anti-rabbit IgG (1:5,000; Thermo Scientific, USA) followed by treatment with Super Signal West Pico chemiluminescent 
substrate (Thermo Scientific, USA). Protein bands were detected by a LAS-3000 image analyzer (Fujifilm, Barcelona, Spain), and the protein levels were quantified by densitometry using Image Gauge Version 4.0 software (Fujifilm). Data were normalized to $\beta$-actin.

\section{Electron microscopy}

For electron microscopy, exosomes were fixed with $2 \%$ paraformaldehyde and loaded on Formvar and carbon-coated copper grids. Then, the grids were placed on $2 \%$ gelatin at $37^{\circ} \mathrm{C}$ for 20 min, rinsed with $0.15 \mathrm{M}$ glycine/PBS, and blocked using $1 \%$ cold water fish-skin gelatin. The grids were viewed using a JEOL 1200EX II transmission electron microscope and imaged using a Gatan digital camera.

\section{Exosome tracer experiment}

To further explore the effect of exosomes on the intestinal tract, an exosome tracer experiment was performed in this study. Exo-CD63-GFP lentivirus was used to fluorescently label CD63 in macrophages. After constructing a stable cell line, CD63-GFP-labeled exosomes were extracted and added to NCM460 intestinal epithelial cells for observation. The exosome tracer experiment was conducted after the cytoskeleton was labeled with phalloidin.

\section{Flow cytometry}

To identify the aforementioned BMDMs and determine their activation level, the cells were harvested and stained with fluorochrome-conjugated antibodies against of the cell-surface antigens F4/80, CD11 b and CD68. BMDMs were collected and incubated with F4/80 antibody (1:60, Abcam, USA), CD11b (1:50, BD Biosciences, USA) and CD68 (1:500, BD Biosciences, USA) for 30 min in the dark at room temperature. Then, BMDMs were centrifuged at $1000 \mathrm{rpm}$ for $5 \mathrm{~min}$ and washed with $1 \mathrm{~mL}$ PBST. Then, the cells were analyzed using fluorescence-activated cell sorting (FACS) (Thermo Fisher Scientific, Waltham, MA, USA). Macrophages are double-positive for $\mathrm{CD} 11 \mathrm{~b}$ and F4/80, and activated macrophages are positive for CD68.

\section{Transepithelial/trans-endothelial electrical resistance (TEER)}

NCM460 cells were seeded $\left(1 \times 10^{5}\right.$ cells/cm2) in a Transwell chamber with $0.4 \mu \mathrm{m}$ pores (Costar, Coring Inc, New York, USA) that had been placed in a 24-well plate. The other Transwell remained blank. After reaching confluence, the cells were differentiated and polarized for 7-10 days in culture medium. TEER was used as a measure of cell monolayer integrity and was assessed before and after all treatments. TEER was measured using an epithelial volt-ohm meter with a chopstick electrode (EMD EVOM2, World Precision Instruments EpithelialVoltohmmete, USA). The electrode was immersed at a $90^{\circ}$ angle with one tip in the basolateral chamber and the other in the apical chamber. Care was taken to prevent electrode contact with the monolayer, and triplicate measurements were recorded for each monolayer. An insert without cells was used as a blank, and its mean resistance was subtracted from the resistance of all samples. TEER $\left(\Omega \mathrm{cm}^{2}\right)=($ Total resistance - Blank resistance $)(\Omega) \star$ Area $\left(\mathrm{cm}^{2}\right)$. 


\section{Statistical analyses}

Data are expressed as the mean \pm standard error of the mean (SEM). One-way analysis of variance (ANOVA), followed by Bonferroni's post-hoc test for repeated measures., was used to analyze the various groups. $\mathrm{P}<0.05$ was considered statistically significant. All authors had access to the study data and had reviewed and approved the final manuscript.

\section{List Of Abbreviations}

IBD: inflammatory bowel disease; DSS: dextran sulfate sodium; BMDMs: murine bone marrow-derived macrophages; LPS: lipopolysaccharide; TMIGD1: transmembrane and immunoglobulin domain containing 1; UC: ulcerative colitis; CD: Crohn's disease; EVs: extracellular vesicles; DAl: disease activity index; NC: negative control; RT-PCR real-time polymerase chain reaction; TNF-a: tumor necrosis factor-a; IL: interleukin; DMEM: Dulbecco's modified Eagle's medium; FACS: fluorescence-activated cell sorting; TEER: transepithelial/transendothelial electrical resistance; AB-PAS: Alcian blue-periodic acid-Schiff ;SEM: standard error of the mean; ANOVA: one-way analysis of variance. AB-PAS: Alcian blue-periodic acidSchiff; FITC-D: Fluorescein Isothiocyanate-Dextran.

\section{Declarations}

\section{Ethics approval and consent to participate}

Collection and analysis of human IBD samples was carried out according to the principles of the Declaration of Helsinki and approved by the Medical Ethics Committee in Shanghai Changhai Hospital, Shanghai, China. Written informed consent was obtained from all of the participants before enrolment. All the animal procedures were approved and conducted in accordance with the guidelines of the Animal Care Committee of Navy Medical University, Shanghai, China.

\section{Consent to publish}

The Author confirms:

that the work described has not been published before; that it is not under consideration for publication elsewhere; that its publication has been approved by all co-authors, if any; that its publication has been approved (tacitly or explicitly) by the responsible authorities at the institution where the work is carried out.

The Author agrees to publication in the Journal indicated below and also to publication of the article in English by Springer in Springer's corresponding English-language journal.

\section{Availability of data and materials}


All data are available on request. The data underlying this article will be shared on reasonable request to the coresponding author.

\section{Competing interests}

Declarations of interest: none

\section{Funding}

Yu Bai is supported by the National Natural Science Foundation of China (No. 81873546 and 82170567 , China), the "Shu Guang" project of Shanghai Municipal Education Commission and Shanghai Education Development Foundation (No. 19SG30, China), the National Key R\&D Program of China (No. 2018YFC1313103, China) and 234 Discipline Climbing Plan of Changhai Hospital, Naval Medical University (No. 2019YXK004, China). Shu-Ling Wang is supported by the National Natural Science Foundation of China (No. 82100587, China), the Shanghai Sailing Program (No. 21YF1458700, China) and the Youth Star-tup Fund of Changhai Hospital, Naval Medical University (No. 2019QNB02, China).

\section{Authors' Contributions}

We thank Xin Chang and Yi-Hang Song for performing the majority of the experiments, analyzing the data and writing the manuscript; Sheng-Bing Zhao for performing some animal experiments, analyzing the data and writing the manuscript; Zi-Xuan He for performing some animal experiments and analyzing some data; Jia-Yi Wu and Lun Gu for performing some cell experiments; Zhao-Shen Li and Can Xu for designing the experiments, supervising the study and performing manuscript review and editing; Shu-Ling Wang for formulating the hypothesis, supervising the study, and performing manuscript review and editing; and Yu Bai for formulating the hypothesis, designing experiments, and performing manuscript review and editing.

\section{Acknowledgements}

The authors thank Dr. Fang Xue for training and improving the organoids cultivating skills, Dr. Xia tian for part intestinal sample collection.

\section{References}

1. Geboes K: Pathology of inflammatory bowel diseases (IBD): variability with time and treatment. Colorectal Dis 2001, 3(1):2-12.

2. Patel $D$, Trivedi $C$, Khan N: Management of Anemia in Patients with Inflammatory Bowel Disease (IBD). Curr Treat Options Gastroenterol 2018, 16(1):112-128.

3. Lord AR, Simms LA, Hanigan K, Sullivan R, Hobson P, Radfordsmith GL: Protective effects offor IBD are related to thepositive strain. 2018. 
4. Larabi A, Barnich N, Autophagy HTTNJ: New insights into the interplay between autophagy, gut microbiota and inflammatory responses in IBD. 2019, 16(5):1-14.

5. de Souza HSP, Fiocchi C, lliopoulos D: The IBD interactome: an integrated view of aetiology, pathogenesis and therapy. Nat Rev Gastroenterol Hepatol 2017, 14(12):739-749.

6. Ananthakrishnan AN, Bernstein CN, lliopoulos D, Macpherson A, Neurath MF, Ali RAR, Vavricka SR, Fiocchi C: Environmental triggers in IBD: a review of progress and evidence. Nat Rev Gastroenterol Hepatol 2018, 15(1):39-49.

7. Angelo Santino AS, Stefania De Santis, Maria De Benedictis, Giovanna Giovinazzo, Chieppa M: Gut Microbiota Modulation and Anti-Inflammatory Properties of Dietary Polyphenols in IBD: New and Consolidated Perspectives. Current Pharmaceutical Design 2017:-.

8. Lissner D, Schumann M, Batra A, Kredel LI, Kühl AA, Erben U, May C, Schulzke JD, Siegmund B: Monocyte and M1 Macrophage-induced Barrier Defect Contributes to Chronic Intestinal Inflammation in IBD. Inflamm Bowel Dis 2015, 21(6):1297-1305.

9. Wang H, Chao K, Ng S, Bai A, Yu Q, Yu J, Li M, Cui Y, Chen M, Hu J et al: Pro-inflammatory miR-223 mediates the cross-talk between the IL23 pathway and the intestinal barrier in inflammatory bowel disease. Genome biology 2016, 17:58.

10. Satsu H, Ishimoto Y, Nakano T, Mochizuki T, Iwanaga T, Shimizu M: Induction by activated macrophage-like THP-1 cells of apoptotic and necrotic cell death in intestinal epithelial Caco-2 monolayers via tumor necrosis factor-alpha. Exp Cell Res 2006, 312(19):3909-3919.

11. Hadis U, Wahl B, Schulz O, Hardtke-Wolenski M, Schippers A, Wagner N, Müller W, Sparwasser T, Förster R, Pabst O: Intestinal tolerance requires gut homing and expansion of FoxP3+ regulatory $\mathrm{T}$ cells in the lamina propria. Immunity 2011, 34(2):237-246.

12. Monteleone G, Fantini MC, Onali S, Zorzi F, Sancesario G, Bernardini S, Calabrese E, Viti F, Monteleone I, Biancone $L$ et al: Phase I clinical trial of Smad7 knockdown using antisense oligonucleotide in patients with active Crohn's disease. Mol Ther 2012, 20(4):870-876.

13. Denzer K, Kleijmeer MJ, Heijnen HF, Stoorvogel W, Geuze HJ: Exosome: from internal vesicle of the multivesicular body to intercellular signaling device. J Cell Sci 2000, 113 Pt 19:3365-3374.

14. Johnstone RM, Adam M, Hammond JR, Orr L, Turbide C: Vesicle formation during reticulocyte maturation. Association of plasma membrane activities with released vesicles (exosomes). J Biol Chem 1987, 262(19):9412-9420.

15. Valadi H, Ekström K, Bossios A, Sjöstrand M, Lee J, Lötvall J: Exosome-mediated transfer of mRNAs and microRNAs is a novel mechanism of genetic exchange between cells. Nature cell biology 2007, 9(6):654-659.

16. M S, TH J: The exosome: a multipurpose RNA-decay machine. Trends in biochemical sciences 2008, 33(10):501-510.

17. Butin-Israeli V, Bui T, Wiesolek H, Mascarenhas L, Lee J, Mehl L, Knutson K, Adam S, Goldman R, Beyder $\mathrm{A}$ et al: Neutrophil-induced genomic instability impedes resolution of inflammation and wound healing. The Journal of clinical investigation 2019, 129(2):712-726. 
18. Yin W, Ouyang S, Li Y, Xiao B, Yang H: Immature Dendritic Cell-Derived Exosomes: a Promise Subcellular Vaccine for Autoimmunity. Inflammation 2012, 36(1):232-240.

19. Magnusson MK, Brynjolfsson SF, Dige A, Uronen-Hansson H, Borjesson LG, Bengtsson JL, Gudjonsson S, Ohman L, Agnholt J, Sjovall H et al: Macrophage and dendritic cell subsets in IBD: $\mathrm{ALDH}+$ cells are reduced in colon tissue of patients with ulcerative colitis regardless of inflammation. Mucosal Immunol 2016, 9(1):171-182.

20. L W, Z Y, S W, F W, W C, B Z, D L, J L, H X, X S et al: Schistosoma japonicumExosomes Derived from Dendritic Cells Treated with Soluble Egg Antigen Attenuate DSS-Induced Colitis. Frontiers in pharmacology 2017, 8(undefined):651.

21. Yang R, Liao Y, Wang L, He P, Hu Y, Yuan D, Wu Z, Sun X: Exosomes Derived From M2b Macrophages Attenuate DSS-Induced Colitis. Frontiers in immunology 2019, 10:2346.

22. Hessvik NP, Llorente A: Current knowledge on exosome biogenesis and release. Cell Mol Life Sci 2018, 75(2):193-208.

23. Ismail N, Wang Y, Dakhlallah D, Moldovan L, Agarwal K, Batte K, Shah P, Wisler J, Eubank TD, Tridandapani SJB: Macrophage microvesicles induce macrophage differentiation and miR-223 transfer. 2013, 121(6):984-995.

24. Menendez A, Willing B, Montero M, Wlodarska M, So C, Bhinder G, Vallance B, Finlay B: Bacterial stimulation of the TLR-MyD88 pathway modulates the homeostatic expression of ileal Paneth cell adefensins. Journal of innate immunity 2013, 5(1):39-49.

25. Ip W, Hoshi N, Shouval D, Snapper S, Medzhitov R: Anti-inflammatory effect of IL-10 mediated by metabolic reprogramming of macrophages. Science (New York, NY) 2017, 356(6337):513-519.

26. De Schepper S, Verheijden S, Aguilera-Lizarraga J, Viola M, Boesmans W, Stakenborg N, Voytyuk I, Schmidt I, Boeckx B, Dierckx de Casterlé I et al: Self-Maintaining Gut Macrophages Are Essential for Intestinal Homeostasis. Cell 2018, 175(2):400-415.e413.

27. Liu R, Tang A, Wang X, Chen X, Zhao L, Xiao Z, Shen S: Inhibition of IncRNA NEAT1 suppresses the inflammatory response in IBD by modulating the intestinal epithelial barrier and by exosomemediated polarization of macrophages. Int J Mol Med 2018, 42(5):2903-2913.

28. Saha S, Aranda E, Hayakawa Y, Bhanja P, Atay S, Brodin N, Li J, Asfaha S, Liu L, Tailor Y et al: Macrophage-derived extracellular vesicle-packaged WNTs rescue intestinal stem cells and enhance survival after radiation injury. Nature communications 2016, 7:13096.

29. Zhang P, Lim S, Jiang K, Chew T, Low B, Lim C: Distinct mRNAs in Cancer Extracellular Vesicles Activate Angiogenesis and Alter Transcriptome of Vascular Endothelial Cells. Cancers 2021, 13(9).

30. Ying W, Riopel M, Bandyopadhyay G, Dong Y, Olefsky JMJC: Adipose Tissue Macrophage-Derived Exosomal miRNAs Can Modulate InVivo and InVitro Insulin Sensitivity. 2017, 171(2):372.

31. Liu L, Yao J, Li Z, Zu G, Feng D, Li Y, Qasim W, Zhang S, Li T, Zeng H et al: miR-381-3p knockdown improves intestinal epithelial proliferation and barrier function after intestinal ischemia/reperfusion injury by targeting nurr1. Cell Death Dis 2018, 9(3):411. 
32. Zahm A, Hand N, Tsoucas D, Le Guen C, Baldassano R, Friedman J: Rectal microRNAs are perturbed in pediatric inflammatory bowel disease of the colon. Journal of Crohn's \& colitis 2014, 8(9):11081117.

33. Othumpangat S, Lindsley W, Beezhold D, Kashon M, Burrell C, Mubareka S, Noti J: Differential Expression of Serum Exosome microRNAs and Cytokines in Influenza A and B Patients Collected in the 2016 and 2017 Influenza Seasons. Pathogens (Basel, Switzerland) 2021, 10(2).

34. Goldie B, Dun M, Lin M, Smith N, Verrills N, Dayas C, Cairns M: Activity-associated miRNA are packaged in Map1b-enriched exosomes released from depolarized neurons. Nucleic acids research 2014, 42(14):9195-9208.

35. Kalluri R, LeBleu VS: The biology, function, and biomedical applications of exosomes. Science 2020, 367(6478).

36. Valadi H, Ekström K, Bossios A, Sjöstrand M, Lee JJ, Lötvall JO: Exosome-mediated transfer of mRNAs and microRNAs is a novel mechanism of genetic exchange between cells. Nat Cell Biol 2007, 9(6):654-659.

37. Fleshner M, Crane CR: Exosomes, DAMPs and miRNA: Features of Stress Physiology and Immune Homeostasis. Trends Immunol 2017, 38(10):768-776.

38. Admyre C, Johansson SM, Qazi KR, Filen JJ, Lahesmaa R, Norman M, Neve EP, Scheynius A, Gabrielsson S: Exosomes with immune modulatory features are present in human breast milk. J Immunol 2007, 179(3):1969-1978.

39. Xiao, Li, Lingying, Liu, Jing, Yang, Yonghui, Yu, Jiake, Chai: Exosome Derived From Human Umbilical Cord Mesenchymal Stem Cell Mediates MiR-181c Attenuating Burn-induced Excessive Inflammation.

40. Bunggulawa EJ, Wang W, Yin T, Wang N, Durkan C, Wang Y, Wang G: Recent advancements in the use of exosomes as drug delivery systems. J Nanobiotechnology 2018, 16(1):81.

41. Zheng X, Chen F, Zhang Q, Liu Y, You P, Sun S, Lin J, Chen N: Salivary exosomal PSMA7: a promising biomarker of inflammatory bowel disease. Protein Cell 2017, 8(9):686-695.

42. Wang H, Ye C, Wu Y, Yang P, Chen C, Liu Z, Wang X: Exosomes in Inflammatory Bowel Disease: What Have We Learned So Far? Curr Drug Targets 2020, 21(14):1448-1455.

43. Ying W, Tseng A, Chang RC, Morin A, Brehm T, Triff K, Nair V, Zhuang G, Song H, Kanameni S et al: MicroRNA-223 is a crucial mediator of PPARy-regulated alternative macrophage activation. J Clin Invest 2015, 125(11):4149-4159.

44. Vian L, Di Carlo M, Pelosi E, Fazi F, Santoro S, Cerio A, Boe A, Rotilio V, Billi M, Racanicchi S et al: Transcriptional fine-tuning of microRNA-223 levels directs lineage choice of human hematopoietic progenitors. Cell death and differentiation 2014, 21(2):290-301.

45. Li M, Zhao J, Cao M, Liu R, Chen G, Li S, Xie Y, Xie J, Cheng Y, Huang L et al: Mast cells-derived MiR223 destroys intestinal barrier function by inhibition of CLDN8 expression in intestinal epithelial cells. Biol Res 2020, 53(1):12.

46. Neudecker V, Haneklaus M, Jensen O, Khailova L, Masterson JC, Tye H, Biette K, Jedlicka P, Brodsky KS, Gerich ME et al: Myeloid-derived miR-223 regulates intestinal inflammation via repression of the 
NLRP3 inflammasome. J Exp Med 2017, 214(6):1737-1752.

47. Zhang D, Lee H, Wang X, Groot M, Sharma L, Dela Cruz CS, Jin Y: A potential role of microvesiclecontaining miR-223/142 in lung inflammation. Thorax 2019, 74(9):865-874.

48. Huppertz C, Jager B, Wieczorek G, Engelhard P, Oliver SJ, Bauernfeind FG, Littlewood-Evans A, Welte T, Hornung V, Prasse A: The NLRP3 inflammasome pathway is activated in sarcoidosis and involved in granuloma formation. Eur Respir J 2020, 55(3).

49. Calvente CJ, Tameda M, Johnson CD, Del Pilar H, Lin YC, Adronikou N, De Mollerat Du Jeu X, Llorente C, Boyer J, Feldstein AE: Neutrophils contribute to spontaneous resolution of liver inflammation and fibrosis via microRNA-223. J Clin Invest 2019, 129(10):4091-4109.

50. Neudecker V, Brodsky KS, Clambey ET, Schmidt EP, Packard TA, Davenport B, Standiford TJ, Weng T, Fletcher AA, Barthel $L$ et al: Neutrophil transfer of miR-223 to lung epithelial cells dampens acute lung injury in mice. Sci Transl Med 2017, 9(408).

51. Li M, Zhao J, Cao M, Liu R, Chen G, Li S, Xie Y, Xie J, Cheng Y, Huang L et al: Mast cells-derived MiR223 destroys intestinal barrier function by inhibition of CLDN8 expression in intestinal epithelial cells. Biological research 2020, 53(1):12.

52. Dietz C, Muller M, Reinhold AK, Karch L, Schwab B, Forer L, Vlckova E, Brede EM, Jakubietz R, Uceyler $\mathrm{N}$ et al: What is normal trauma healing and what is complex regional pain syndrome I? An analysis of clinical and experimental biomarkers. Pain 2019, 160(10):2278-2289.

53. Zabana Y, Loren V, Domenech E, Aterido A, Garcia-Jaraquemada A, Julia A, Vicario M, Pedrosa E, Ferreiro M, Troya $\mathrm{J}$ et al: Transcriptomic identification of TMIGD1 and its relationship with the ileal epithelial cell differentiation in Crohn's disease. Am J Physiol Gastrointest Liver Physiol 2020, 319(2):G109-G120.

54. Ranjan A, lyer SV, Iwakuma T: Suppressive roles of A3AR and TMIGD3 i1 in osteosarcoma malignancy. Cell Cycle 2017, 16(10):903-904.

55. Arafa E, Bondzie PA, Rezazadeh K, Meyer RD, Hartsough E, Henderson JM, Schwartz JH, Chitalia V, Rahimi N: TMIGD1 is a novel adhesion molecule that protects epithelial cells from oxidative cell injury. Am J Pathol 2015, 185(10):2757-2767.

56. Cattaneo E, Laczko E, Buffoli F, Zorzi F, Bianco MA, Menigatti M, Bartosova Z, Haider R, Helmchen B, Sabates-Bellver $\mathrm{J}$ et al: Preinvasive colorectal lesion transcriptomes correlate with endoscopic morphology (polypoid vs. nonpolypoid). EMBO Mol Med 2011, 3(6):334-347.

57. Zabana Y, Lorén V, Domènech E, Aterido A, Garcia-Jaraquemada A, Julià A, Vicario M, Pedrosa $E$, Ferreiro M, Troya $\mathrm{J}$ et al: Transcriptomic identification of TMIGD1 and its relationship with the ileal epithelial cell differentiation in Crohn's disease. Am J Physiol Gastrointest Liver Physiol 2020, 319(2):G109-g120.

\section{Figures}




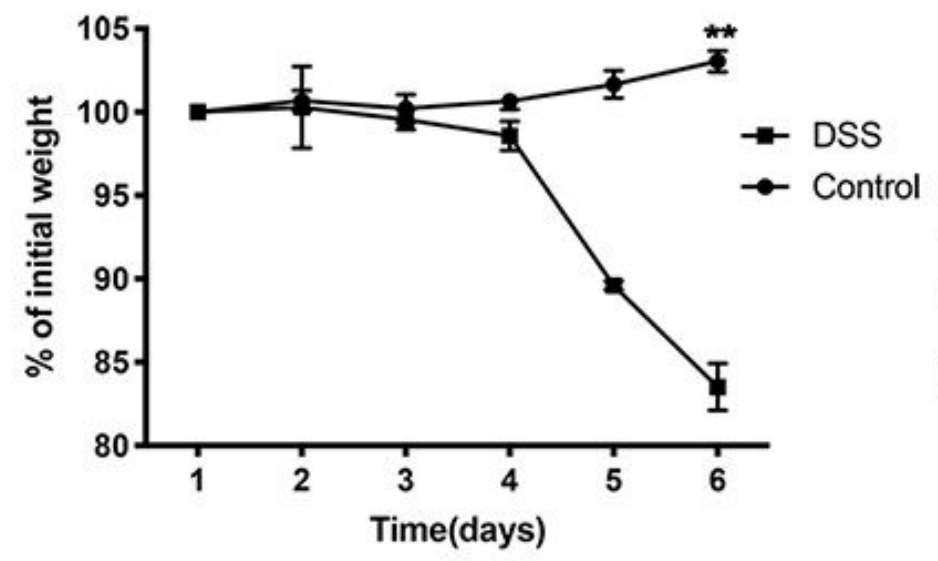

C
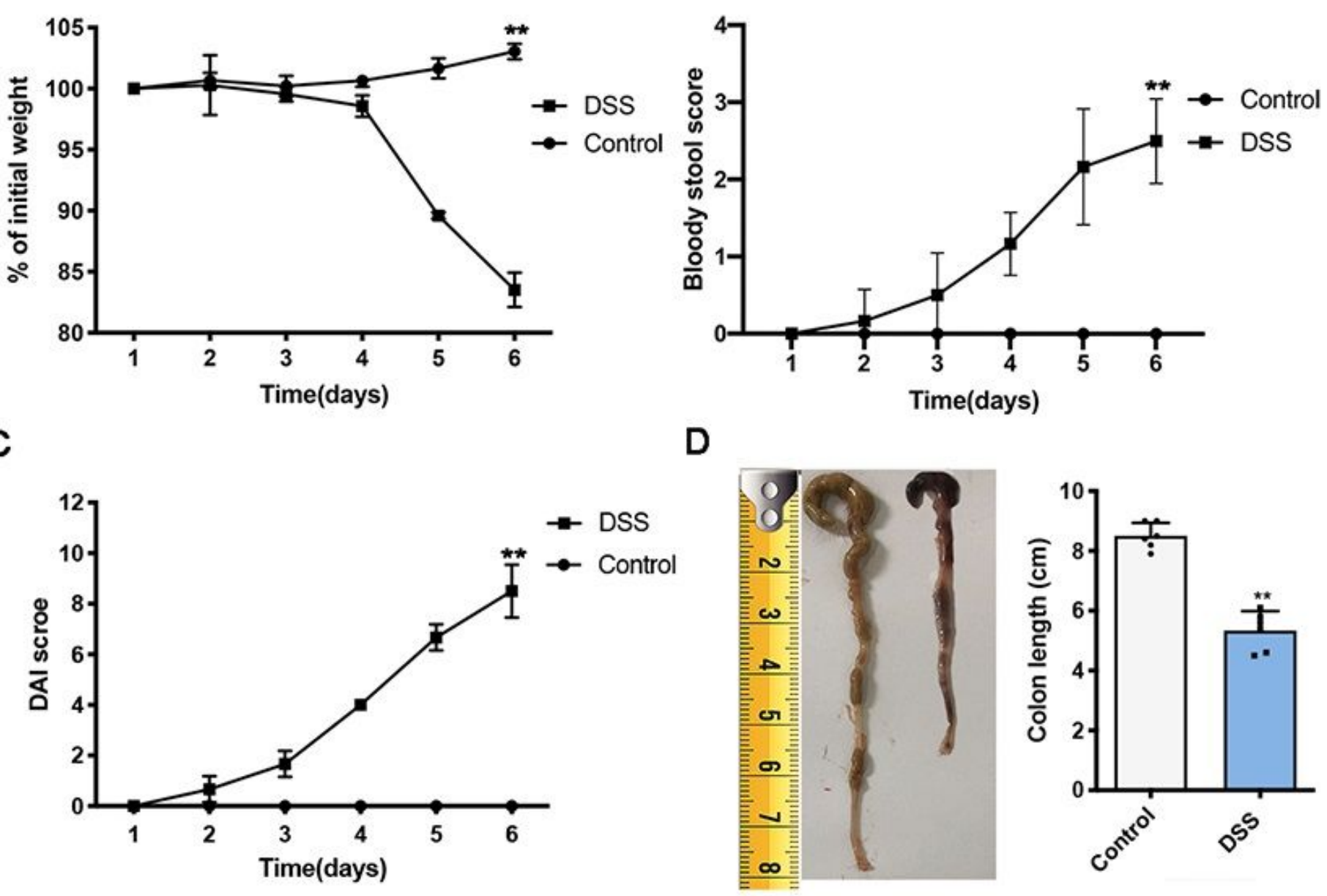

D
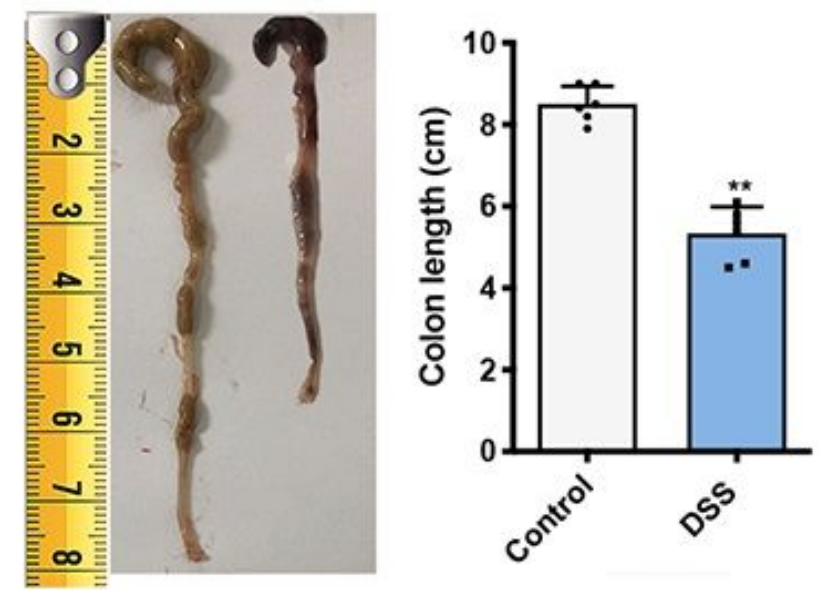

\section{Figure 1}

Induction and symptoms of the IBD mouse model with DSS. DSS (3\%) was given to C57BL/ 6 mice for 6 days to induce the IBD mouse model, while water was given to the control group. Body weight loss, stool consistency and occult or gross blood per rectum and colon length were recorded by two researchers who were blinded to the treatments. (A) Compared to the control group, the mice in the DSS group exhibited a significant decrease in body weight ( $n=6$ per group). (B-C) The bloody stool and DAI scores were obviously elevated in the DSS group ( $n=6$ per group). (D) Moreover, there was a significant shortening of colon length in DSS-induced mice compared to the control group ( $n=6$ per group). ${ }^{*} P<0.01$ vs. the control group. 
A

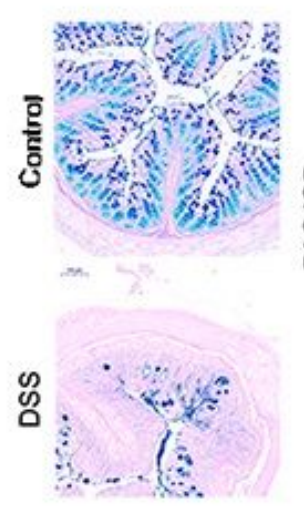

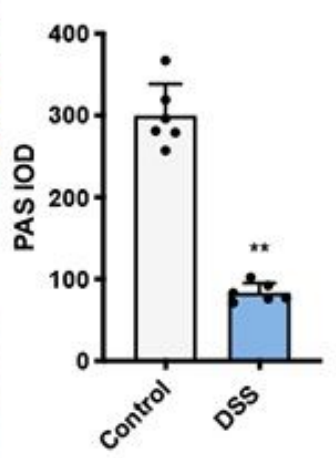

B

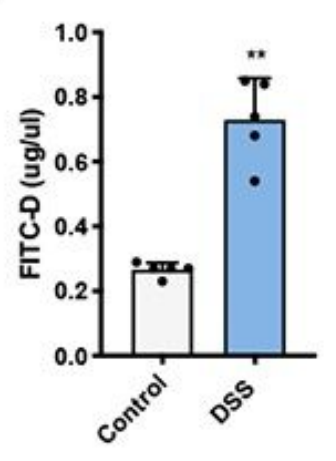

C

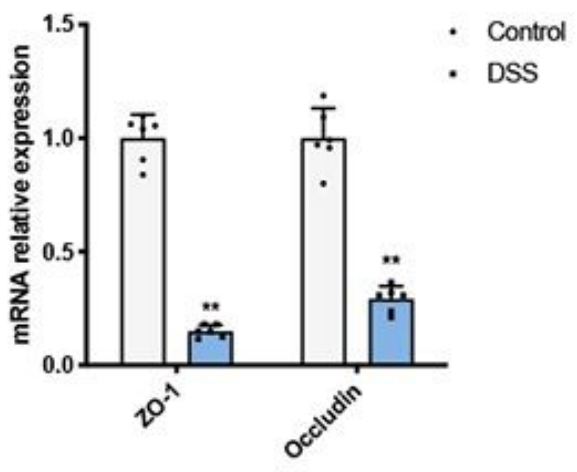

D

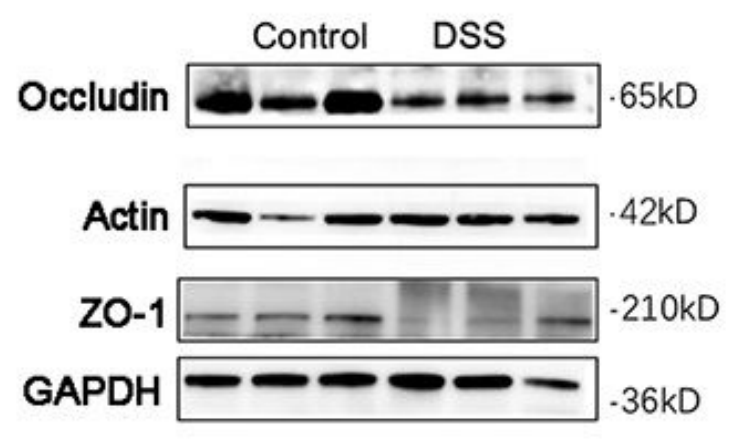

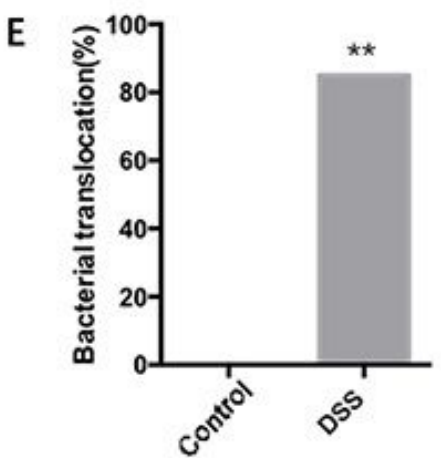

\section{Figure 2}

The intestinal mucosal barrier was impaired in DSS-induced IBD mice. C57BL/6 mice received DSS (3\%) for 6 days, and the control group was given water. (A) The goblet cell count was analyzed by Alcian Blueperiodic acid-Schiff (AB-PAS) staining, and the results showed a significant decrease in the DSS group (n $=6$ per group). ${ }^{\star \star} P<0.01$ vs. the control group. (B) The integrity of the intestinal mucosal barrier was explored with FITC-D and flora translocation experiments. Compared to the control group, DSS-induced mice showed an obvious elevation in FITC-D ( $n=5$ per group). ${ }^{* *} P<0.01$ vs. the control group. (C-D) The intestinal mucosal barrier-specific markers ZO- 1 and Occludin were detected by RT-PCR R ( $\mathrm{n}=6$ per group) and Western blotting ( $\mathrm{n}=3$ per group), and their expression was significantly decreased in IBD mice. (E) The bacterial translocation rate was much higher in the DSS group, which illustrated that the integrity of the intestinal mucosal barrier was impaired ( $n=20$ per group). ${ }^{* *} P<0.01$ vs. the control group. 
A

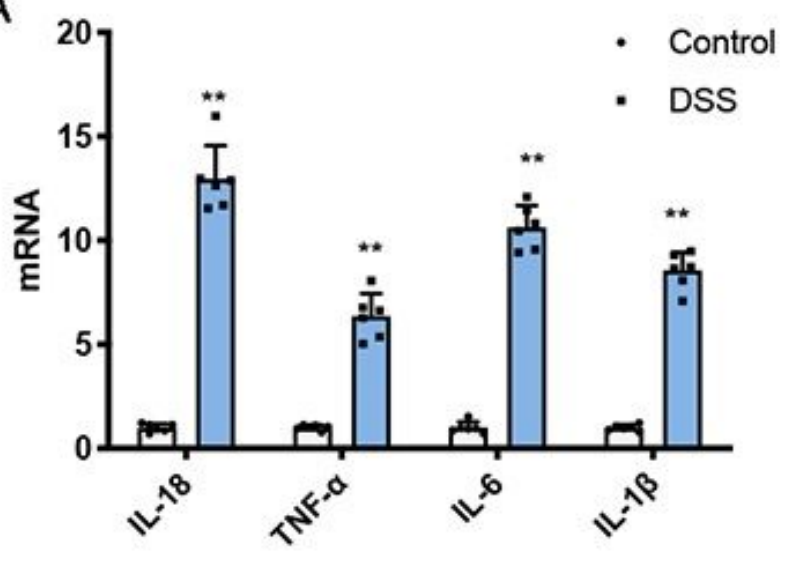

B
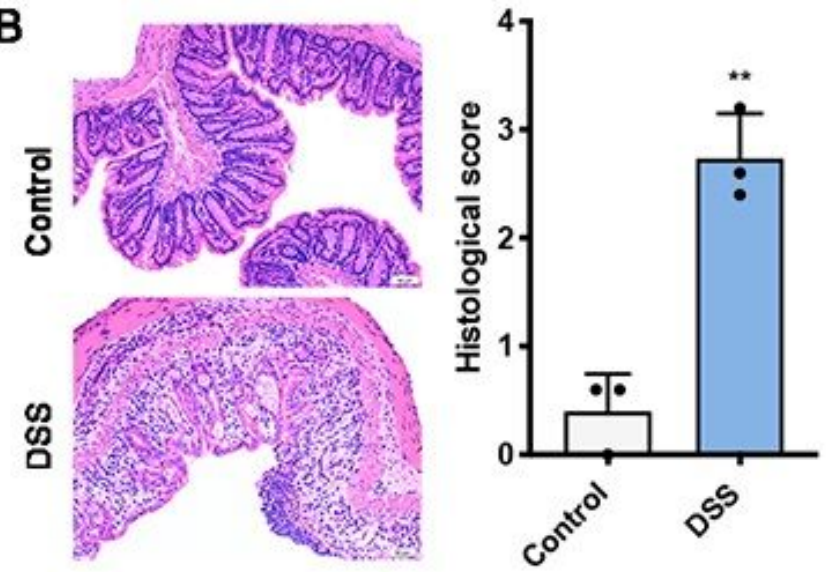

C

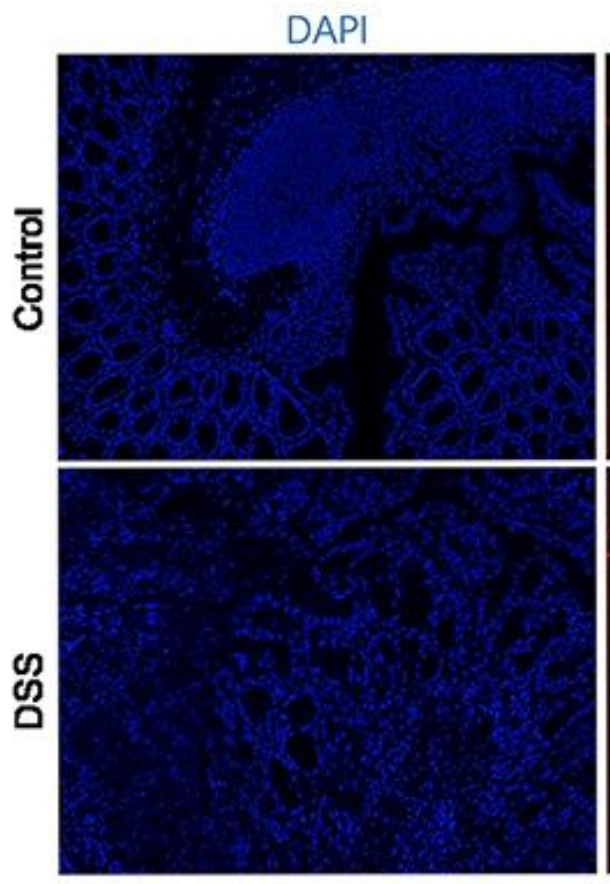

$\mathrm{F} 4 / 80$

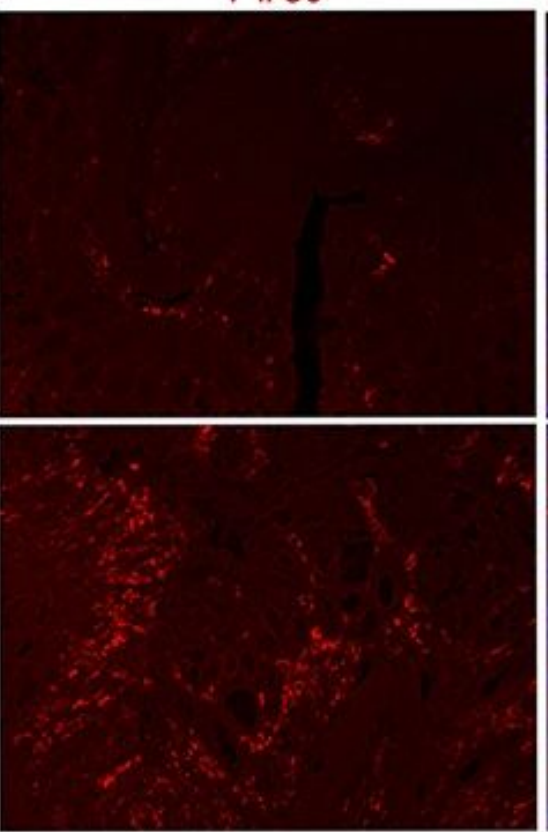

Merge

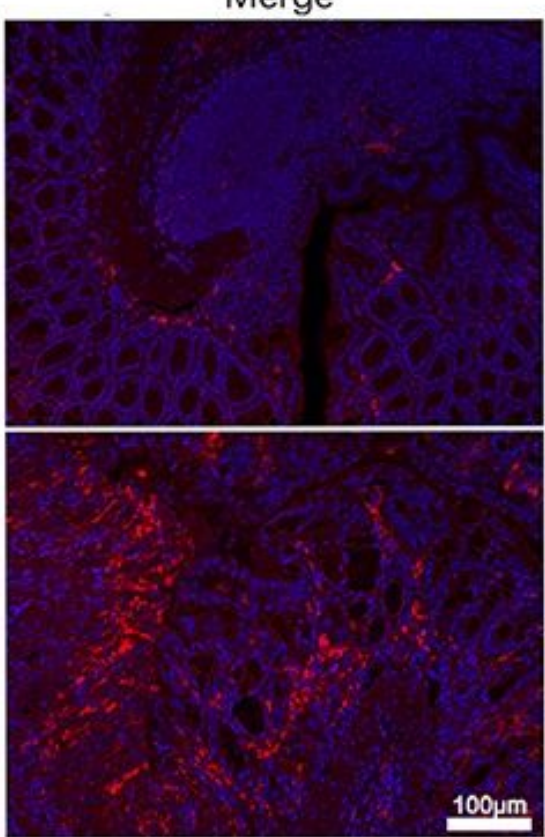

\section{Figure 3}

Macrophages were activated during DSS-induced colitis. C57BL/ 6 mice received DSS (3\%) for 6 days, and the control group was given water. (A) The inflammatory factors IL-18, TNF- $a$, IL- 6 and IL-1 $\beta$ were analyzed by RT-PCR. The mice in the DSS group showed enhanced levels of IL-18, TNF-a, IL-6 and IL-1 $\beta$ in intestinal tissue ( $n=6$ per group). ${ }^{\star \star} P<0.01$ vs. the control group. (B) Colon sections were examined with hematoxylin and eosin (H\&E) (20x). Compared to the control group, the mice in the DSS group showed aggravated inflammatory infiltration in the left colon tissue. ${ }^{* \star} \mathrm{P}<0.01$ vs. the control group. (C) The macrophage count was detected by immunofluorescence with the specific marker F4/80. The middle edge of the left colon was further detected by immunofluorescence staining, and compared to the control group, the mice in the DSS group showed enhanced macrophage activation in the epithelium. 
A

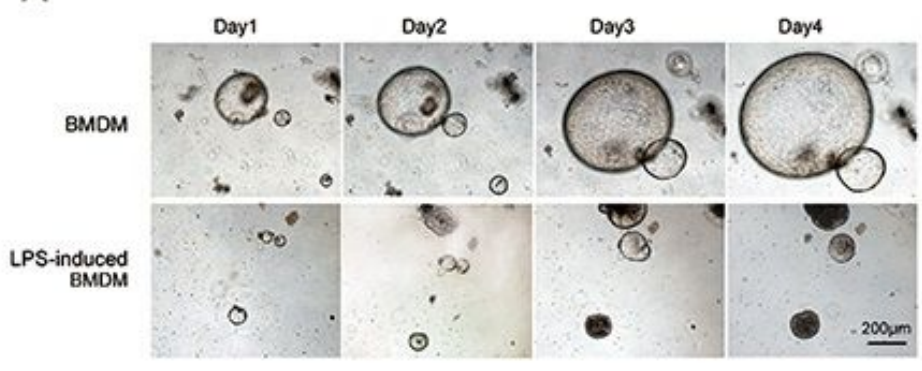

C

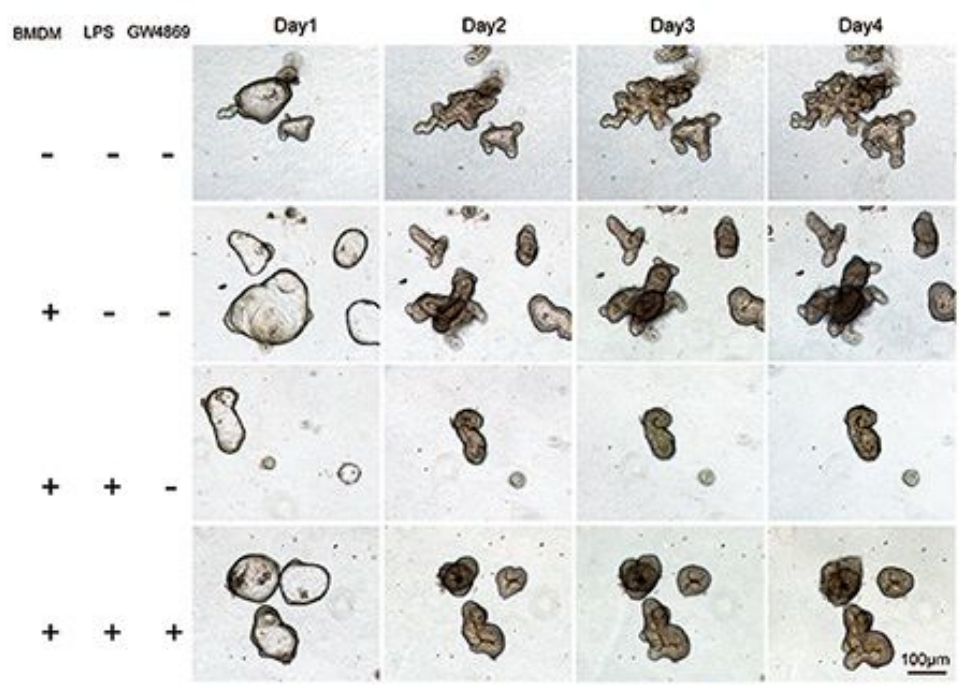

B

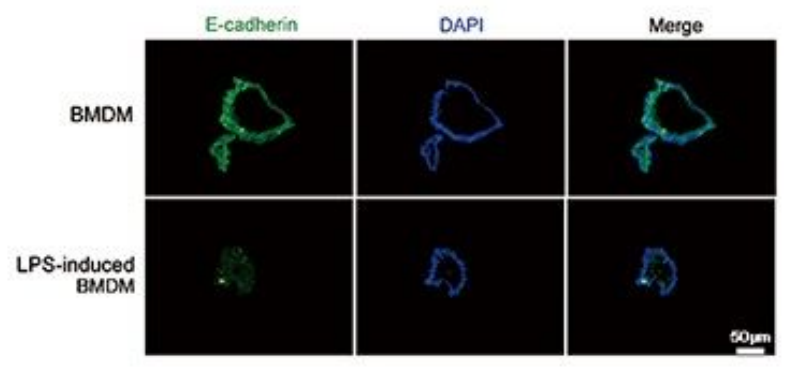

D

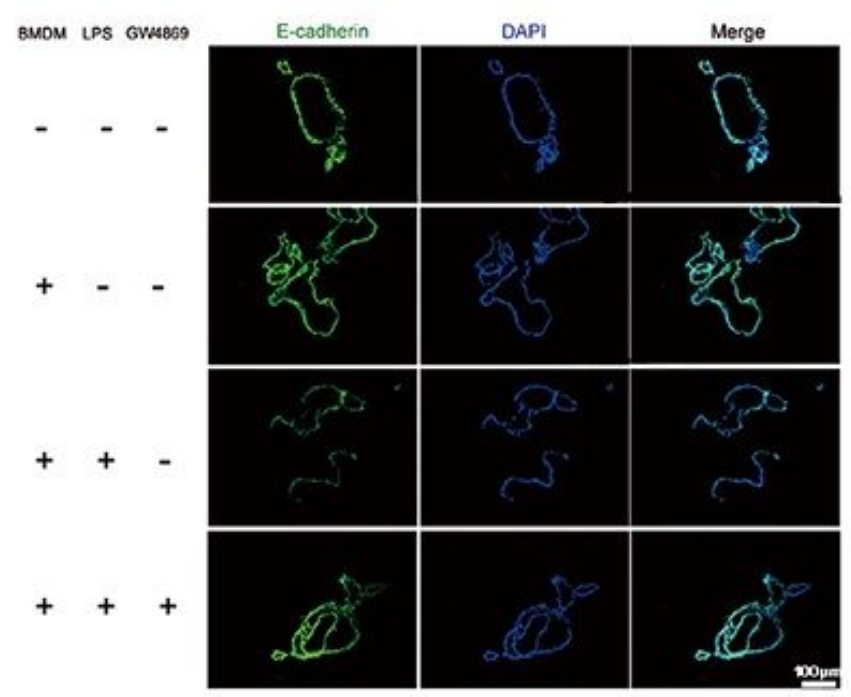

Figure 4

Colonic organoids from mice and humans were affected by exosomes derived from macrophages. The Transwell system was used to coculture macrophages with mouse and human organoids, with or without LPS. The exosome inhibitor GW4869 (GW, $40 \mu \mathrm{M})$ was also applied for $72 \mathrm{~h}$ in a Transwell system. Ecadherin detection by immunofluorescence staining was performed to observe the barriers of colonic organoids. (A) Colonic organoids from mice were cocultured with macrophages. Compared to the plain BMDM group, the development of mouse organoids was restricted in the LPS-induced BMDM group. (B) Compared to the BMDM group, the mouse organoids in the LPS-induced BMDM group showed a decrease in E-cadherin. (C) Colonic organoids from humans cocultured with macrophages and GW4869 cells were used to analyze the effect of exosomes. Growth was not significantly different between the BMDM(-) LPS(-) GW4869(-) group and the BMDM(+) LPS(-) GW4869(-) group. Compared to the BMDM(+)LPS(-)GW4869(-) group, the human organoids in the BMDM(+) LPS(+) GW4869(-) group, in which BMDMs were activated by LPS, showed significant growth restriction, similar to those from mice. In addition, compared to the BMDM(+) LPS(-) GW4869(-) group, the human organoids in the BMDM(+) LPS(+) GW4869(+) group treated with the exosome inhibitor showed recovery of the growth trend. (D) The intestinal barrier was detected with the mucosal barrier-specific marker E-cadherin, and the 
expression of E-cadherin not significantly different between the BMDM(-) LPS(-) GW4869(-) group and the BMDM(+) LPS(-) GW4869(-) group. Compared to the BMDM(+) LPS(-) GW4869(-) group, the expression of E-cadherin in human organoids in the BMDM(+) LPS(+) GW4869(-) group was significantly decreased, similar to those from mice. Compared to the BMDM(+) LPS(+) GW4869(-) group, the expression of Ecadherin in human organoids in the BMDM(+) LPS(+) GW4869(+) group, in which an exosome inhibitor was added, was significantly increased.

A

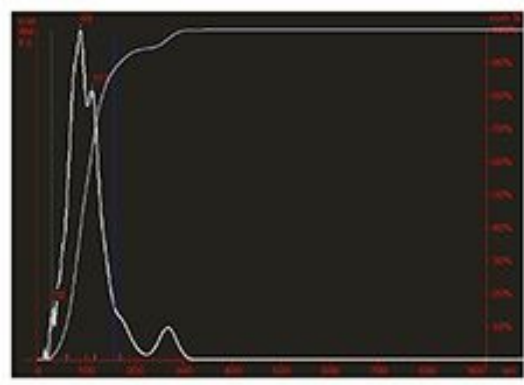

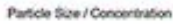

C

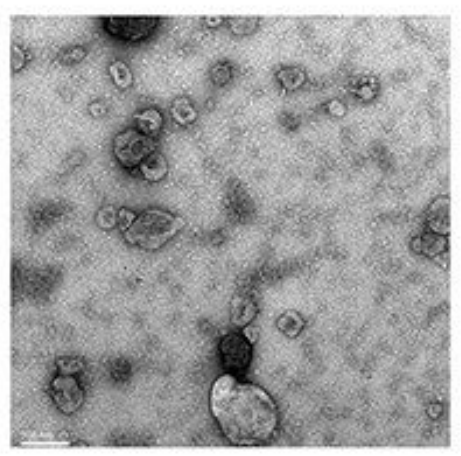

B

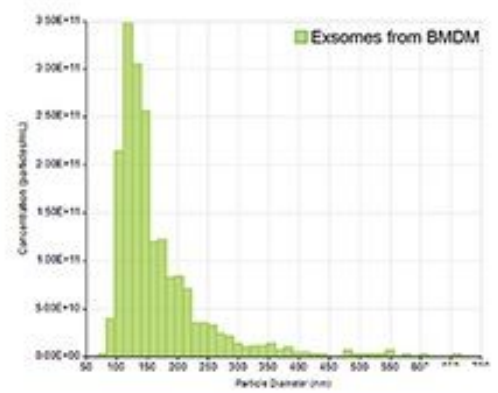

D

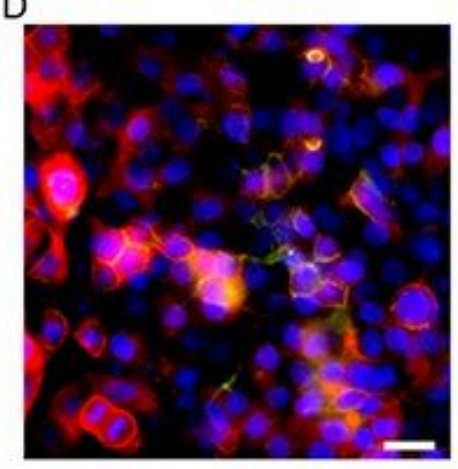

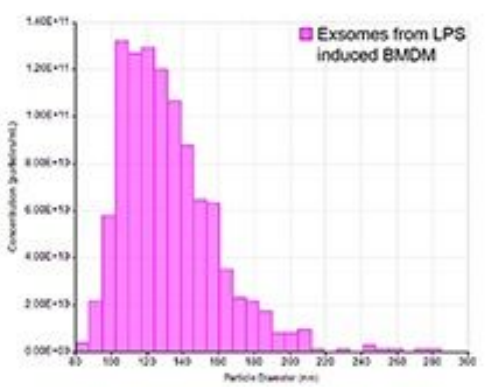

E

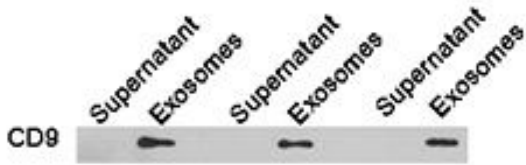

F

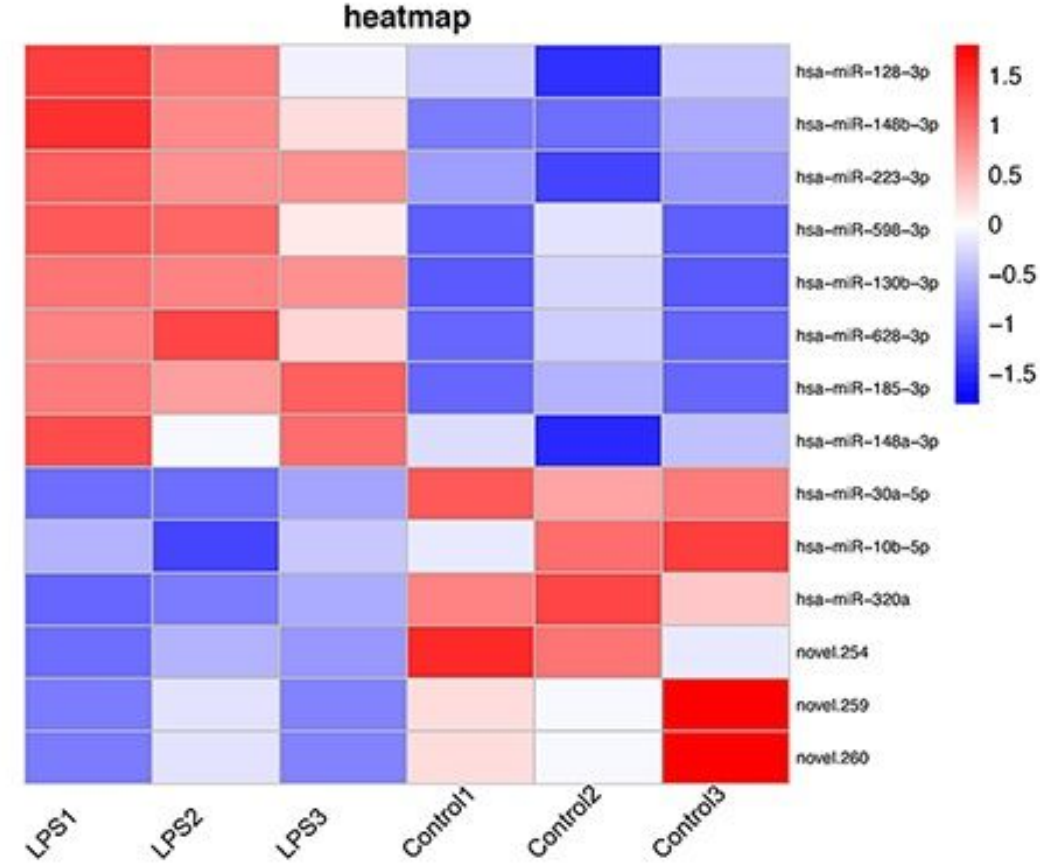

G

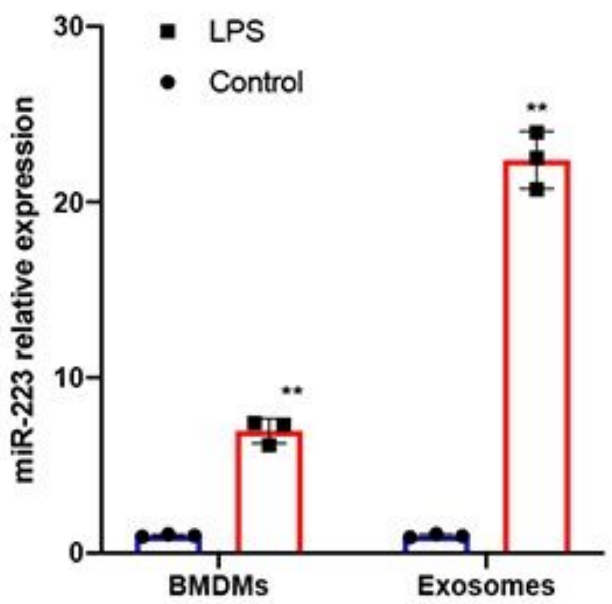


Exosomes were purified from the supernatant of macrophages, and miRNA sequencing analysis was performed. (A) The size distribution profile of exosomes was analyzed by a NanoSight instrument, and the diameter of isolated exosomes mainly ranged from $90 \mathrm{~nm}$ to $120 \mathrm{~nm}$. (B) The exosome concentration in each group was analyzed by the NTA system, and the exosome concentration in the LPS group was higher than that of the nonactivated BMDMs. (C) Exosomes were analyzed by negative-staining transmission electron microscopy (TEM), and the morphology of isolated exosomes was in accordance with that reported in the literature. Scale bar, $200 \mathrm{~nm}$. (D) Macrophages were transfected with CD63-GFP so that exosomes with GFP could be bioautographed by NCM460 cytoskeleton-labeled red fluorescence. Ex vivo fluorescence imaging was conducted to determine the distribution of GFP-labeled exosomes in enterocytes. (E) The exosome-specific markers CD9 was detected by Western blotting, and we found increased expression of these markers in exosomes compared to the supernatant. (F) Differentially expressed microRNAs were screened by high-throughput sequencing analysis, and the expression of specific microRNAs was verified in both macrophages and exosomes ( $n=3$ per group). The 10 most significantly upregulated and downregulated miRNAs are listed, including miR-223. (G) The expression of miR-223 in the LPS group was six times greater than that in the control group ( $n=3$ per group). ${ }^{\star \star} \mathrm{P}<$ 0.01 vs. the BMDM group. 
A

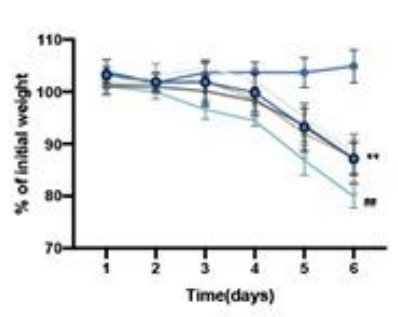

D

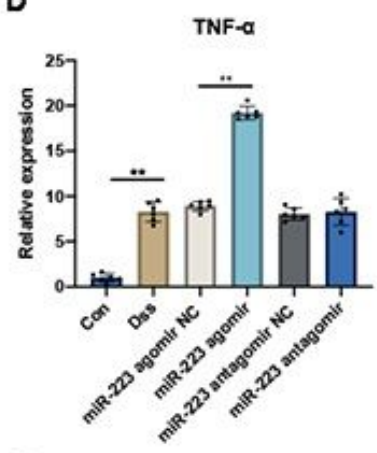

E
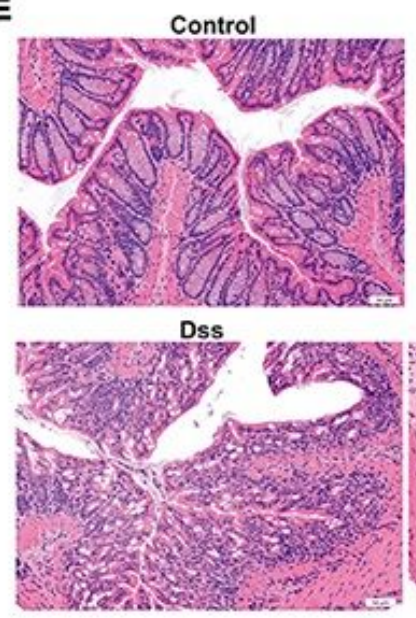

B

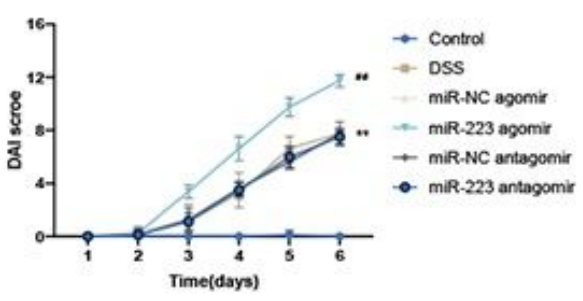

C
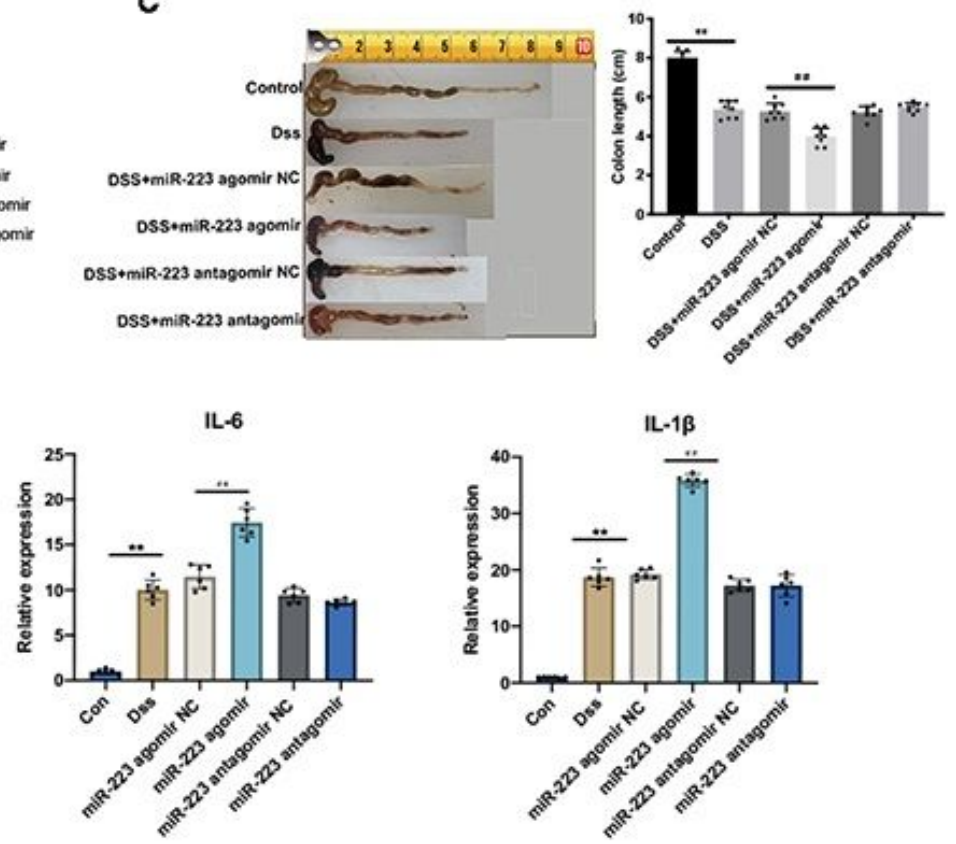
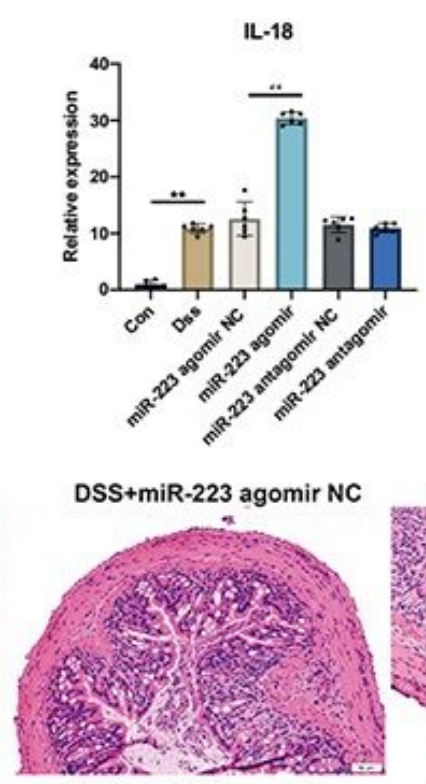

DSS+miR-223 agomir

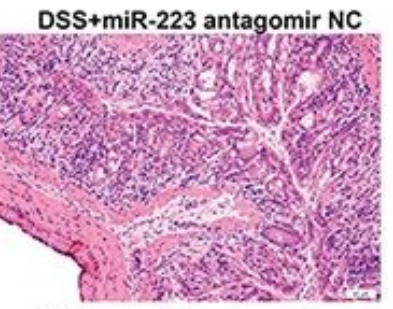

DSS+miR-223 antagomir
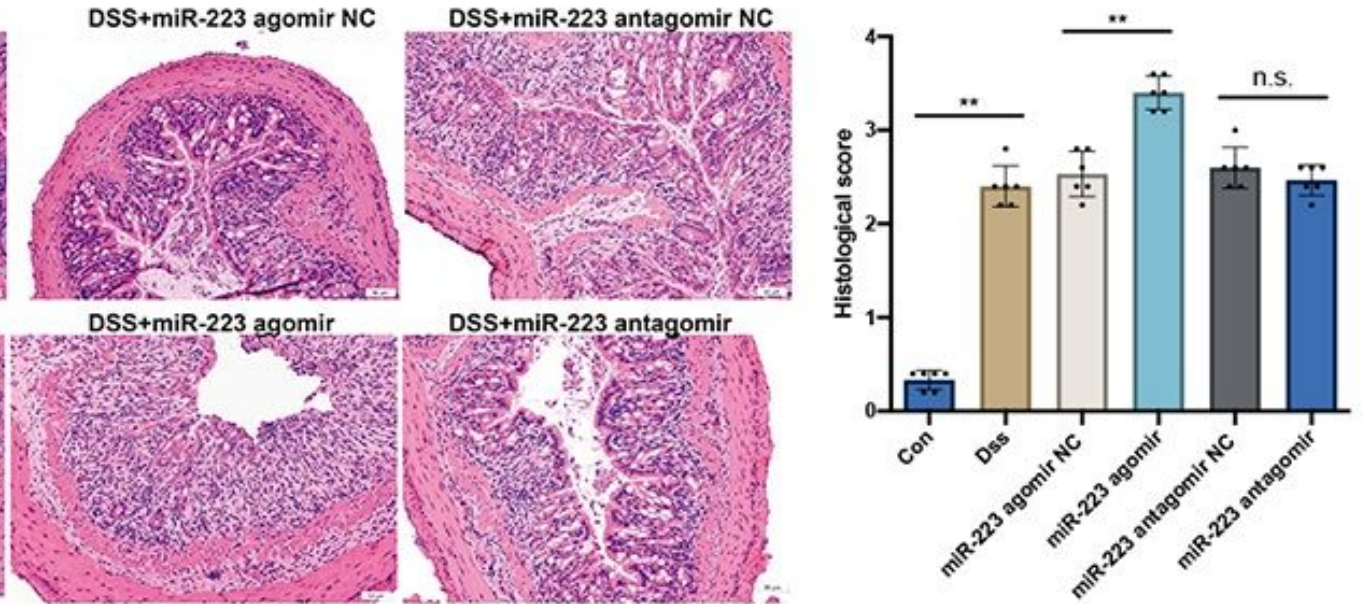

\section{Figure 6}

miR-223 in exosomes contributes to the increase in inflammation in IBD mice. C57BL/6 mice received DSS (3\%) for 6 days, and the control group was given water. DSS+exosomesmiR-223 agomir NC mice were administered DSS and injected with miR-223 agomir NC exosomes $(108,0.1 \mathrm{ml}$, i.p. injection, once a day, from day 2 to 6), DSS+exosomesmiR-223 agomir mice were administered DSS and injected with miR-223 agomir exosomes (108, $0.1 \mathrm{ml}$, i.p. injection, once a day, from day 2 to 6$)$, DSS+exosomesmiR223 antagomir NC mice were administered DSS and injected with miR-223 antagomir NC exosomes (108, $0.1 \mathrm{ml}$, i.p. injection, once a day, from day 2 to 6), and DSS+exosomesmiR-223 antagomir mice were administered DSS and injected with miR-223 antagomir exosomes (108, 0.1ml, i.p. injection, once a day, from day 2 to 6). (A-C) The body weight, DAl score and colon length were determined by two investigators blinded to the treatment groups. Compared to the control group, the mice in the DSS group exhibited significant deterioration of colon length, body weight and DAl score, while the injection of exosomal miR223 largely aggravated the inflammation caused by IBD ( $n=8$ per group). ** $P<0.01$ vs. the control 
group. \#\# $\mathrm{P}<0.01$ vs. the DSS+exosomesmiR-223 agomir NC group. (D) The proinflammatory factors were analyzed in different groups by RT-PCR, and the levels of TNF-a, IL-18, IL- 6 and IL-1b indicated aggravated colonic inflammation in the DSS group compared to the control group. Furthermore, injection of exosomes with the miR-223 agomir exacerbated inflammatory factor infiltration ( $n=6$ per group). ** $P$ $<0.01$ vs. the control group. \#\# $P<0.01$ vs. the DSS+exosomesmiR-223 agomir NC group. (E) Histopathological changes in the colons of mice treated with macrophage exosomes were evaluated by H\&E staining. Compared to the control group mice, the mice in the DSS group showed aggravated inflammatory infiltration in the left colon, while injection of exosomes with the miR-223 agomir further aggravated inflammation ( $n=6$ per group). ${ }^{* *} P<0.01$ vs. the control group. \#\# $P<0.01$ vs. the DSS+exosomesmiR-223 agomir NC group. 


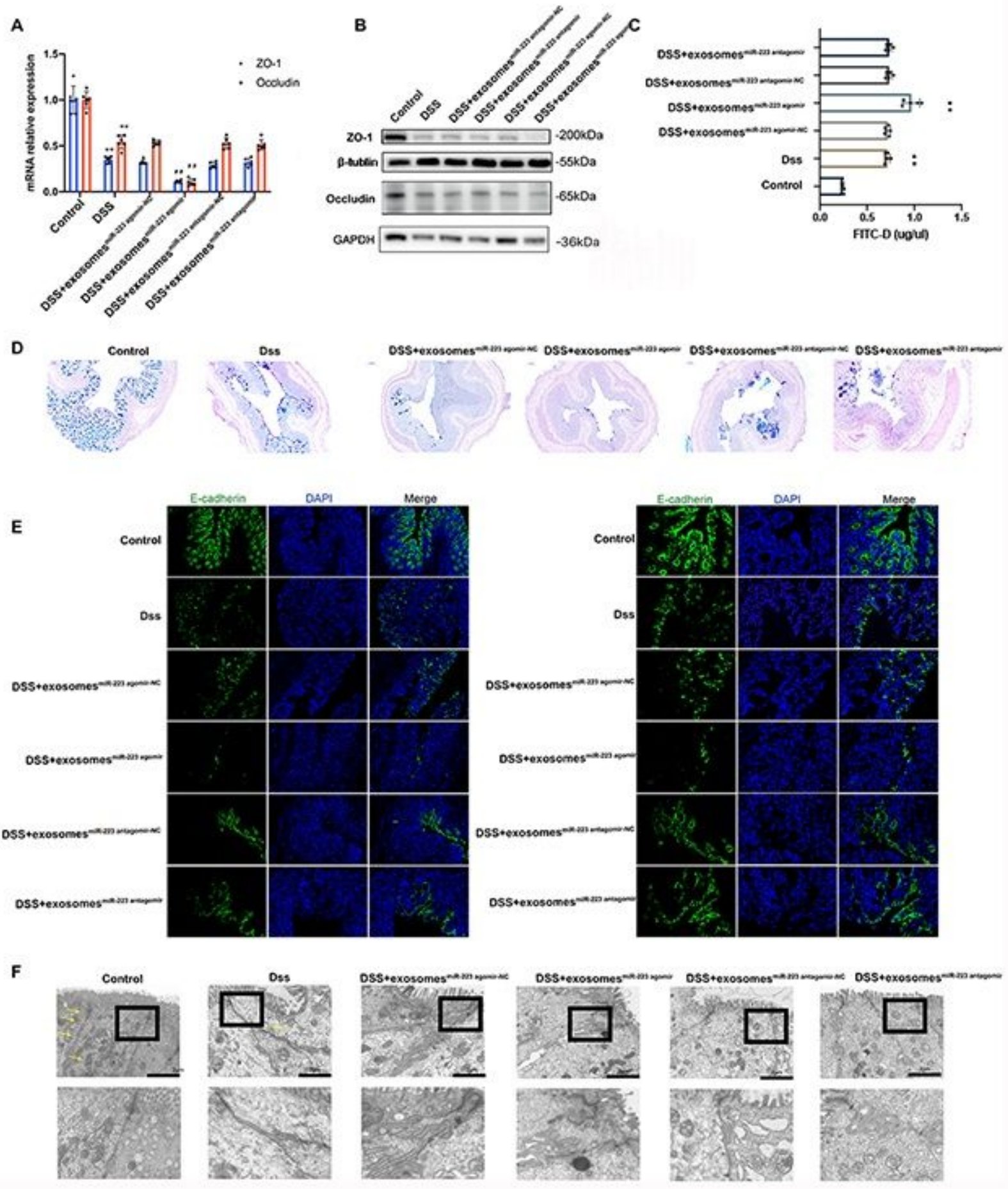

\section{Figure 7}

The integrity of the intestinal barrier was significantly disrupted by administration of exosomes containing high levels of miR-223 in the left colon of IBD mice. C57BL/ 6 mice received DSS (3\%) for 6 days, and the control group was given water. DSS+exosomesmiR-223 agomir NC mice were administered DSS and injected with miR-223 agomir NC exosomes $(108,0.1 \mathrm{ml}$, i.p. injection, once a day, from day 2 to 7), DSS+exosomesmiR-223 agomir mice were administered DSS and injected with miR-223 agomir 
exosomes (108, $0.1 \mathrm{ml}$, i.p. injection, once a day, from day 2 to 7), DSS+exosomesmiR-223 antagomir NC mice were administered DSS and injected with miR-223 antagomir-NC exosomes (108, 0.1ml, i.p. injection, once a day, from day 2 to 7), and DSS+exosomesmiR-223 antagomir mice were administered DSS and injected with miR-223 antagomir exosomes $(108,0.1 \mathrm{ml}$, i.p. injection, once a day, from day 2 to 7). (A-B) The specific mucosal barrier factors ZO-1 and Occludin in tissue from mice in the 6 different groups were analyzed by RT-PCR and Western blotting. The left colon was isolated, and the mRNA levels of ZO-1 and Occludin were decreased in the DSS group compared to the control group. Furthermore, injection of exosomes with the miR-223 agomir downregulated intestinal mucosal barrier-related molecules ( $n=6$ per group). ${ }^{\star \star P} P<0.01$ vs. the control group. \#\#P $<0.01$ vs. the $D S S+e x o s o m e s m i R-223$ agomir NC group. (C) Experimental mice were administered $0.6 \mathrm{mg} / \mathrm{g}$ FITC-D four hours before they were euthanized, and the integrity of the intestinal barrier was analyzed by measuring FITC-D in the plasma of mice. Compared to the control group, the concentration of FITC-D in the DSS group was significantly increased. The concentration of FITC-D in the DSS+exosomesmiR-223 agomir group was further increased ( $n=5$ per group). ${ }^{*} \mathrm{P}<0.01$ vs. the control group. \#\#P<0.01 vs. the DSS+exosomesmiR-223 agomir NC group. (D) AB-PAS staining was used to detect mucous secretion in the intestines of mice in different groups. Compared to the control group, the expression of AB-PAS in the DSS group was significantly decreased, and the expression of AB-PAS in the DSS+exosomesmiR-223 agomir group was further decreased. (E) The expression of specific mucosal barrier molecules was further detected by immunofluorescence staining. Compared to the control group, mice in the DSS group showed reduced mucosal barrier expression in the left colon epithelium. Compared to the DSS+exosomesmiR-223 agomir NC group, mice in the DSS+exosomesmiR-223 agomir group showed a further reduction. (F) Colonic samples were prepared for TEM, and the luminal surface of the intestinal mucosa was observed (100x and 500x). Yellow arrows indicate specialized tight junctions and desmosomes. Intestinal disorganization was very obvious in the DSS+exosomesmiR-223 agomir group. 

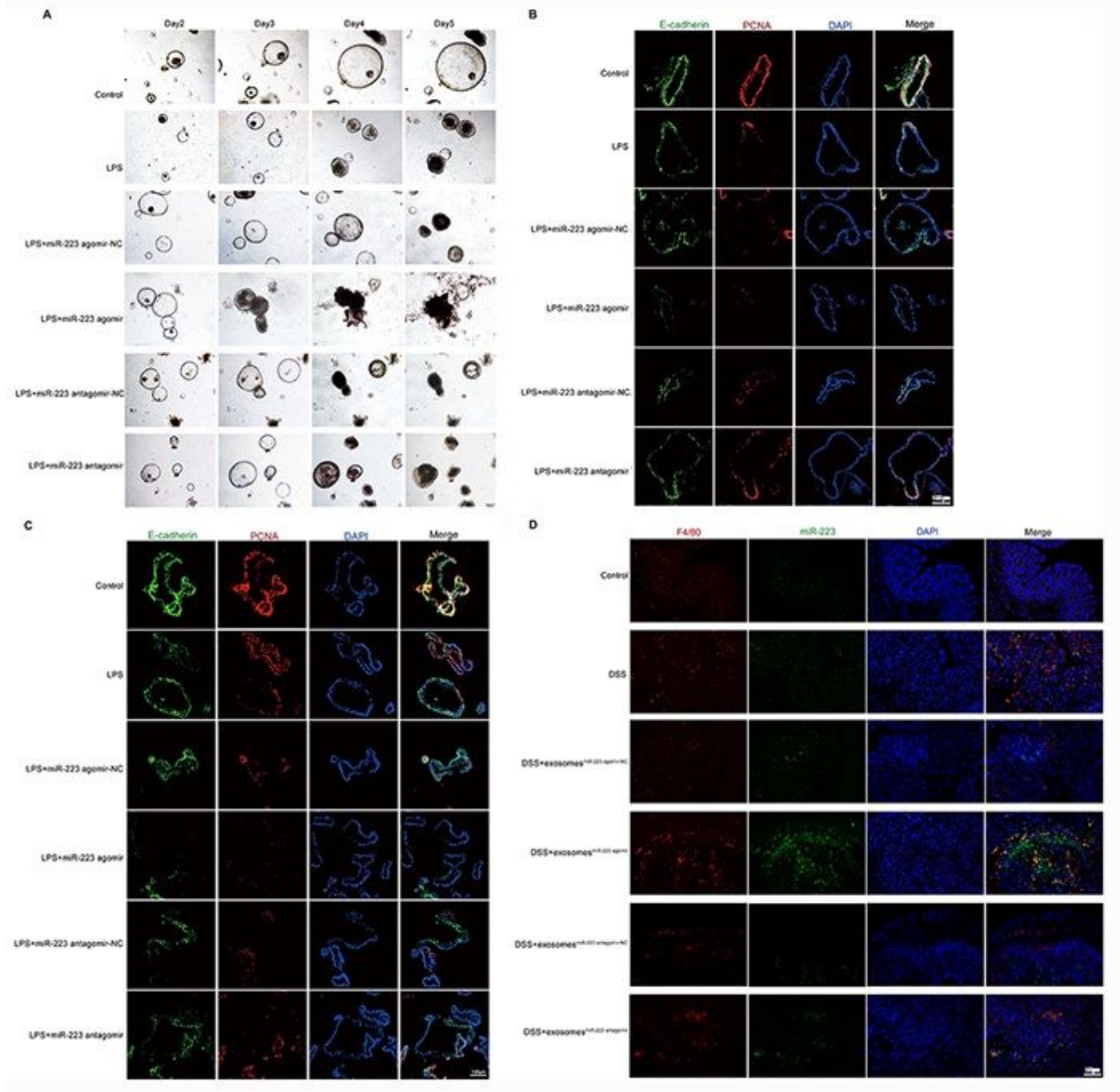

\section{Figure 8}

Exosomes with high miR-223 expression affect intestinal mucosal barrier expression in both mouse and human colonic organoids. Lentivirus was used to establish stable miR-223-knockout, overexpression and corresponding negative control (NC) macrophage strains. After stable transfectant, the miR-223 agomir NC, miR-223 agomir, miR-223 antagomir NC, miR-223 antagomir and control RAW264.7 cell line was activated in refueled LPS (100 ng/ml, every $24 \mathrm{~h}$ ) medium and then cocultured with cells and colon organoids. Specifically, the LPS group colon organoids were cocultured with LPS-induced RAW264.7, the LPS+miR-223 agomir NC group colon organoids were cocultured with miR-223 agomir NC RAW264.7 
which administered LPS, LPS+miR-223 agomir colon organoids were cocultured with miR-223 agomir RAW264.7 treated with LPS, LPS+miR-223 antagomir NC colon organoids were cocultured with miR-223 antagomir NC RAW264.7 stimulated with LPS, and LPS+miR-223 antagomir colon organoids were cocultured with miR-223 antagomir RAW264.7 induced by LPS. The treatment of exosomes on mice were shown as before. (A) Conoids from mice cocultured with macrophages were observed by microscopy, and the development of organoids in the LPS group was restricted compared to that in the control group. Development in the LPS+miR-223 agomir group was further aggravated compared to that in the LPS+miR-223 NC. (B-C) Expression of the proliferation-related molecule PCNA and the intestinal barrierrelated molecule E-cadherin was detected by immunofluorescence in both mouse and healthy human colonoids. The expression of E-cadherin and PCNA in the LPS group was decreased compared to that in the control group, and a further decreasing trend was observed in the LPS+miR-223 agomir group. (D) Proliferation of macrophages was detected by F4/80 and was significantly increased in the DSS+exosomesmiR-223 agomir group compared to the DSS+exosomesmiR-223 agomir NC group; increased miR-223 expression was observed in the mouse intestine. 

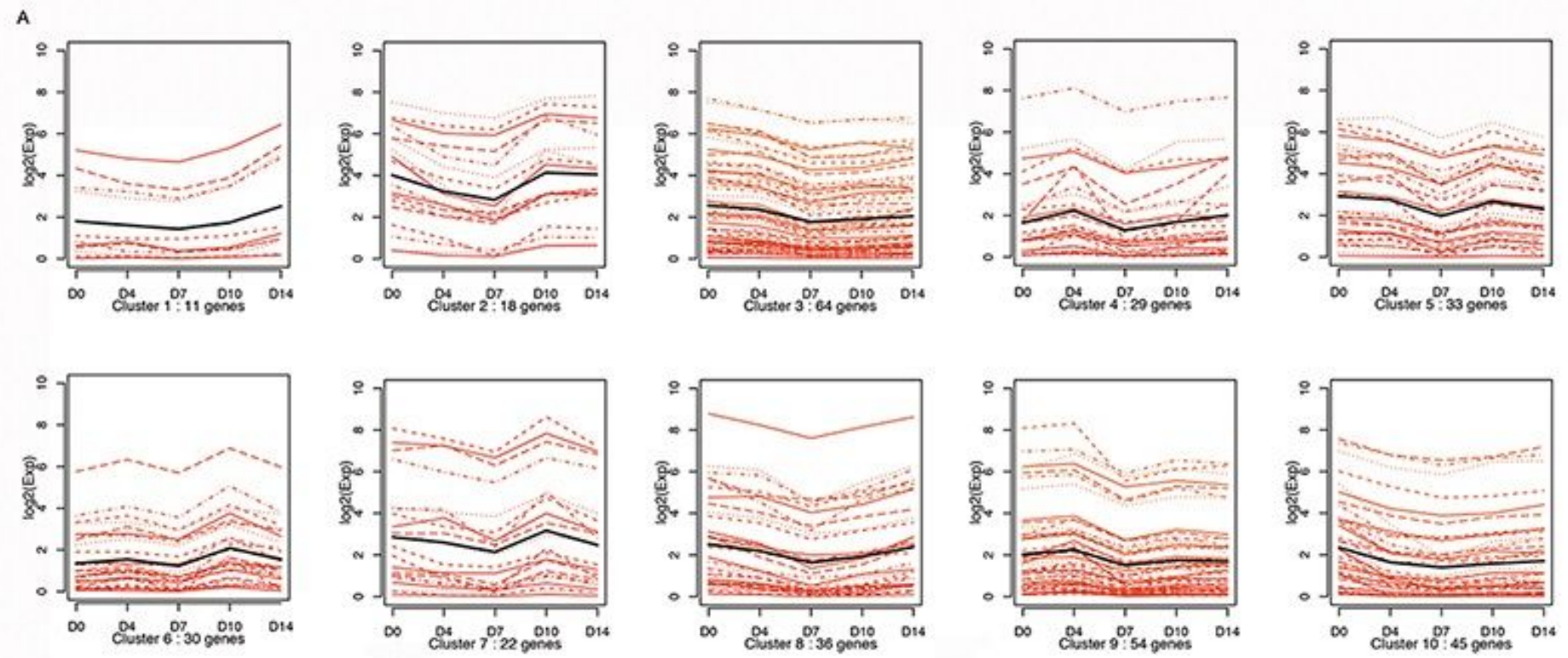
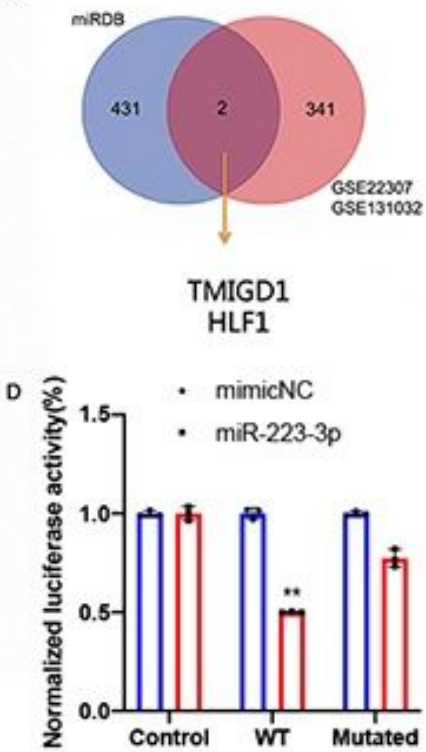

c

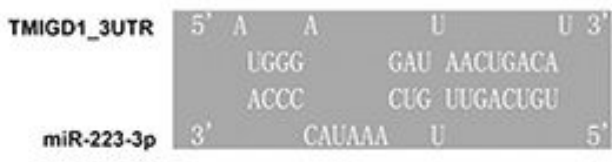

$m f e:-22.0 \mathrm{kcal} / \mathrm{mol}$

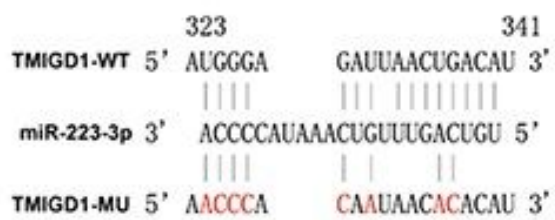

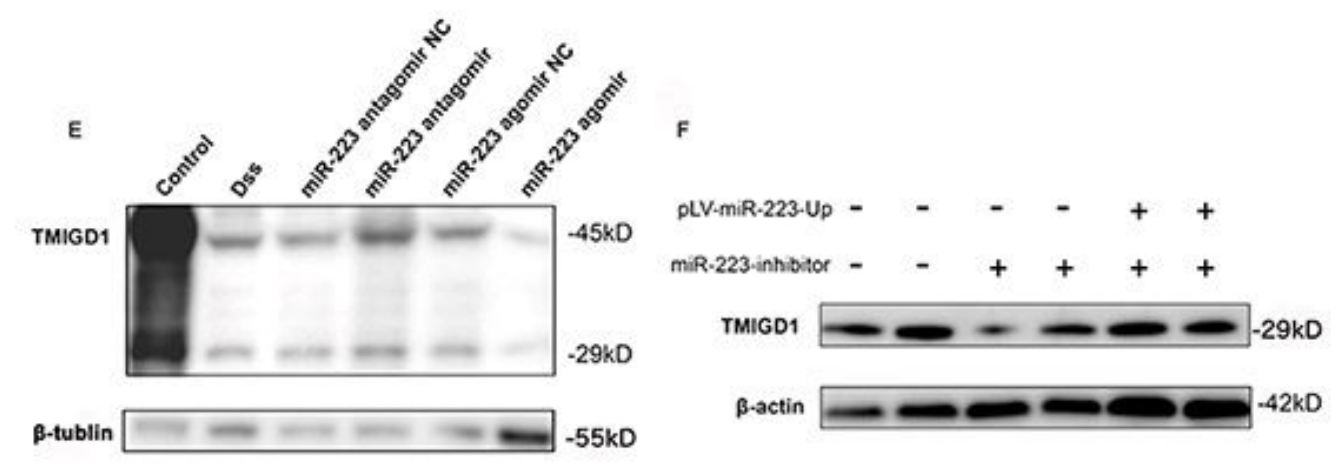

\section{Figure 9}

Macrophage-derived exosomes affect the intestinal mucosal barrier by mediating miR-223 to regulate the downstream target gene TMIGD1. (A-B) Expression of 341 mRNAs in IBD mice according to the timedependent analysis were performed, mRNAs that were predicted to bind to miR-233 were examined to obtain the candidate gene and TMIGD1 was selceted. (C) Prediction of the binding sites of miR-223 and the transcription factor TMIGD1. (D) Dual-luciferase experiments were performed to verify that miR-223 mainly regulates TMIGD1. The luciferase activity was significantly different in cells transfected with wildtype or mutant TMIGD1 and miR-223 mimics or negative control ( $n=3$ per group). ${ }^{* \star} P<0.01$. (E) The expression of TMIGD1 in the DSS+miR-223 agomir group was significantly restricted compared to the DSS+miR-223 agomir-NC group, which was same as the expression trend between control and DSS group. (F) The expression of TMIGD1 in NCM460 cells with miR-223 overexpression or inhibition was 
detected by Western blotting. TMIGD1 expression was decreased in the miR-223 overexpression group, which identified the regulatory function of miR-223 in vitro ( $n=2$ per group).

A
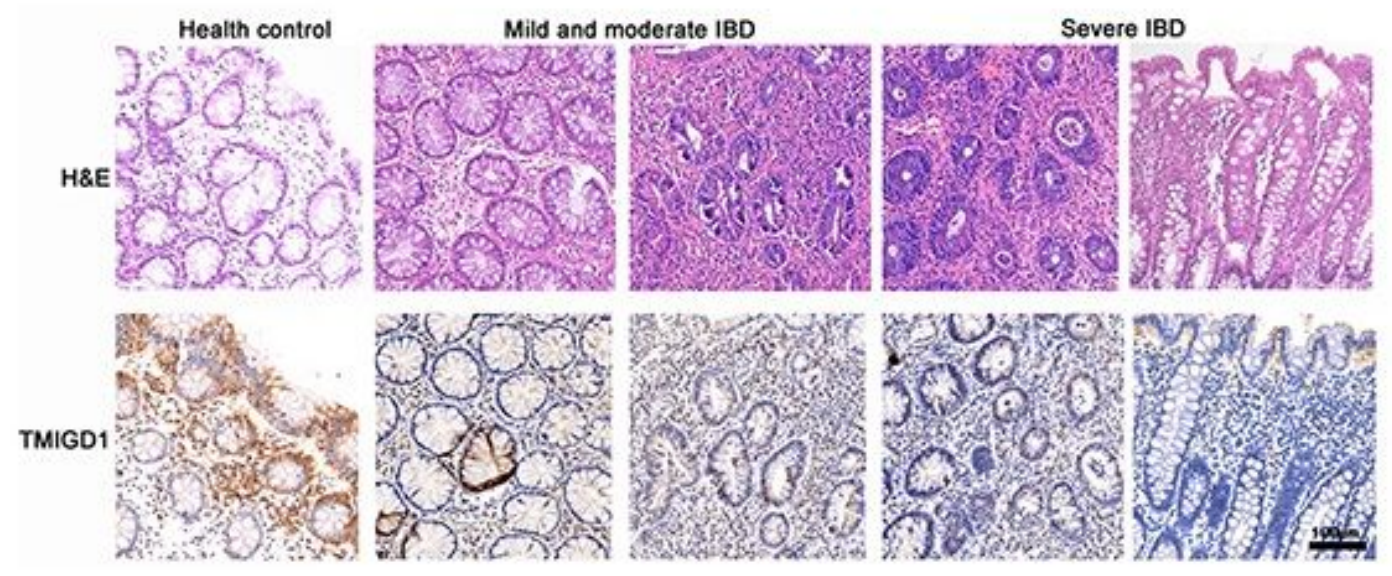

B
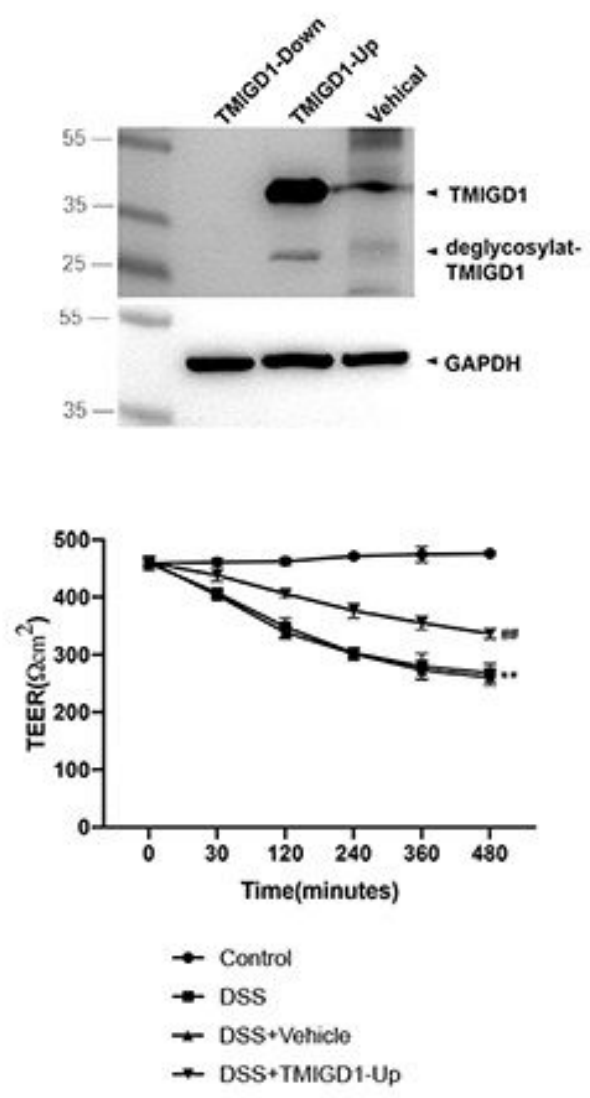

C

DAPI
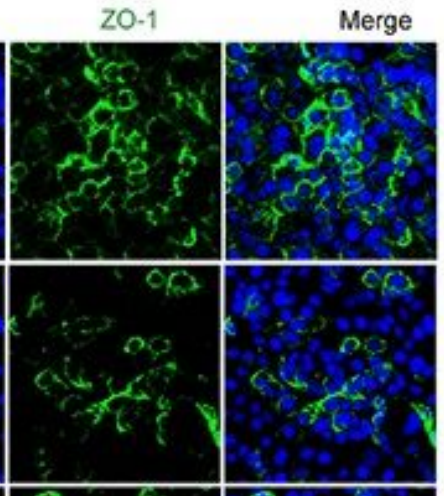

DSS+TMIGD1-Up
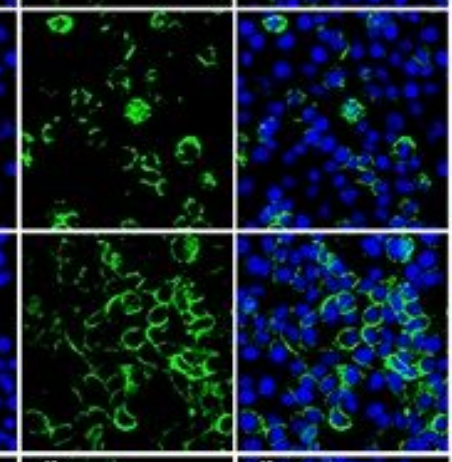

DSS+TMIGD1-Down
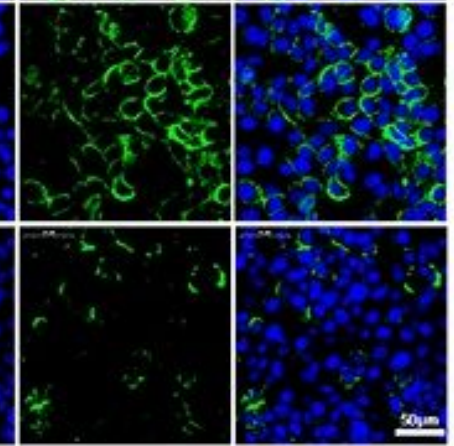

\section{Figure 10}

TMIGD1 can promote the integrity of the mucosal barrier. (A) Protein expression of TMIGD1 in colon tissues from IBD patients and healthy controls was analyzed by immunohistochemistry. The expression of TMIGD1 gradually decreased with increasing disease severity. (B) TMIGD1-Up and TMIGD1-Down 
intestinal epithelial NCM460 cells were used, and the effect of TMIGD1 on the barrier function of NCM460 cells was studied. The transepithelial electric resistance (TEER) was detected to measure the integrity of the intestinal mucus. The resistance value of NCM460 were analyzed $(n=3) * * P<0.01$ vs. the control group. \#\# P $<0.01$ vs. DSS+Vehicle (C) Immunofluorescence was used to detect the expression of intestinal barrier molecules in NCM460 cells. Both the TEER and barrier molecule expression were decreased in the DSS+miR-223 agomir group compared to the negative control group.

\section{Supplementary Files}

This is a list of supplementary files associated with this preprint. Click to download.

- 4supportingdocuments0811.docx 